\title{
SECOND ORDER OPTIMALITY CONDITIONS IN THE SMOOTH CASE AND APPLICATIONS IN OPTIMAL CONTROL*
}

\author{
Bernard Bonnard ${ }^{1}$, Jean-Baptiste Caillau ${ }^{2}$ and Emmanuel Trélat ${ }^{3}$
}

\begin{abstract}
The aim of this article is to present algorithms to compute the first conjugate time along a smooth extremal curve, where the trajectory ceases to be optimal. It is based on recent theoretical developments of geometric optimal control, and the article contains a review of second order optimality conditions. The computations are related to a test of positivity of the intrinsic second order derivative or a test of singularity of the extremal flow. We derive an algorithm called COTCOT (Conditions of Order Two and COnjugate Times), available on the web, and apply it to the minimal time problem of orbit transfer, and to the attitude control problem of a rigid spacecraft. This algorithm involves both normal and abnormal cases.
\end{abstract}

Mathematics Subject Classification. 49K15, 49-04, 70Q05.

Received May 19, 2005.

\section{INTRODUCTION}

Let $M$ (resp., $N$ ) be a smooth manifold of dimension $n$ (resp., $m$ ). Consider on $M$ the control system

$$
\dot{x}(t)=f(x(t), u(t))
$$

where $f: M \times N \longrightarrow T M$ is smooth, and where the controls are bounded measurable functions, defined on intervals $[0, T(u)]$ of $\mathbb{R}^{+}$, and taking their values in a subset $U$ of $N$. Let $M_{0}$ and $M_{1}$ be two subsets of $M$. Denote by $\mathcal{U}$ the set of admissible controls, so that their associated trajectories steer the system from an initial point of $M_{0}$ to a final point in $M_{1}$. For such a control $u$, the cost of the associated trajectory $x(\cdot)$ is defined by

$$
C\left(t_{f}, u\right)=\int_{0}^{t_{f}} f^{0}(x(t), u(t)) \mathrm{d} t
$$

Keywords and phrases. Conjugate point, second-order intrinsic derivative, Lagrangian singularity, Jacobi field, orbit transfer, attitude control.

* Work supported in part by the French Space Agency (contract 02/CNES/0257/00).

${ }^{1}$ Univ. Dijon, IMB, Bât. Mirande, 9 avenue Alain Savary, 21078 Dijon Cedex, France; Bernard.Bonnard@u-bourgogne.fr

2 ENSEEIHT-IRIT, UMR CNRS 5505, 2 rue Camichel, 31071 Toulouse, France; caillau@n7.fr

${ }^{3}$ Univ. Orléans, UFR Sciences Mathématiques, Labo. MAPMO, UMR 6628, Route de Chartres, BP 6759, 45067 Orléans Cedex

2, France; emmanuel.trelat@univ-orleans.fr

(c) EDP Sciences, SMAI 2007 
where $f^{0}: M \times N \longrightarrow \mathbb{R}$ is smooth. We investigate the optimal control problem of determining a trajectory $x(\cdot)$ solution of (1), associated to a control $u$ on $\left[0, t_{f}\right]$, so that $x(0) \in M_{0}, x\left(t_{f}\right) \in M_{1}$, and minimizing the cost $C$. The final time $t_{f}$ may be fixed or not.

The well known Pontryagin maximum principle [24] asserts that, if the trajectory $x(\cdot)$, associated to a control $u \in \mathcal{U}$, is optimal on $\left[0, t_{f}\right]$, then there exists a nonpositive real number $p^{0}$ and an absolutely continuous mapping $p(\cdot)$ on $\left[0, t_{f}\right]$, called adjoint vector, satisfying $\left(p(\cdot), p^{0}\right) \neq(0,0)$ and $p(t) \in T_{x(t)}^{*} M$, such that there holds

$$
\dot{x}(t)=\frac{\partial H}{\partial p}\left(x(t), p(t), p^{0}, u(t)\right), \dot{p}(t)=-\frac{\partial H}{\partial x}\left(x(t), p(t), p^{0}, u(t)\right),
$$

almost everywhere on $\left[0, t_{f}\right]$, where $H\left(x, p, p^{0}, u\right)=\langle p, f(x, u)\rangle+p^{0} f^{0}(x, u)$ is the Hamiltonian, and

$$
H\left(x(t), p(t), p^{0}, u(t)\right)=M\left(x(t), p(t), p^{0}\right)
$$

almost everywhere on $\left[0, t_{f}\right]$, where $M\left(x(t), p(t), p^{0}\right)=\max _{v \in U} H\left(x(t), p(t), p^{0}, v\right)$. If the final time $t_{f}$ is not fixed, there holds moreover $M\left(x(t), p(t), p^{0}\right)=0$, for every $t \in\left[0, t_{f}\right]$. If $M_{0}$ and $M_{1}$ (or just one of both) are regular submanifolds of $M$, then the adjoint vector can be chosen so that $p(0) \perp T_{x(0)} M_{0}$ and $p\left(t_{f}\right) \perp T_{x\left(t_{f}\right)} M_{1}$.

Definition 0.1. A 4-tuple $\left(x(\cdot), p(\cdot), p^{0}, u(\cdot)\right)$ solution of $(3)$ and (4) is called an extremal. An extremal is said to be normal (resp. abnormal) if $p^{0}<0$ (resp. $p^{0}=0$ ). If moreover transversality conditions are satisfied, the extremal is called a $B C$-extremal.

Throughout the article, we will assume that the domain $U$ of values of controls is open in $N$. In this case, the maximization condition (4) implies $\frac{\partial H}{\partial u}\left(x(t), p(t), p^{0}, u(t)\right)=0$. If this condition permits to compute the extremal control as a smooth function, then the extremal is smooth, and we are in the so-called smooth case. In this article, we restrict ourselves to this situation ${ }^{1}$. In particular, the bang-bang case is not treated here, but can however be dealt with using similar geometric constructions (see [2]).

The first conjugate point of a given extremal solution is defined as the first point at which the extremal ceases to be locally optimal (essentially, in topology $L^{\infty}$ ). The objective of this paper is to recall the theoretical framework of the conjugate point theory, and then to provide algorithms of computation, that are implemented on two nontrivial case studies.

The outline of the article is the following. In Section 1, we provide an overview of second order necessary and/or sufficient conditions for optimality, mainly inspired by [3]. In Section 2, we define the concept of conjugate time in a geometric way, using the classical approach by central fields and singularities of Lagrangian manifolds. This geometric characterization can be implemented, and in Section 2 we give some algorithms to compute conjugate times, and we comment on their practical implementation. Section 3 is devoted to describe our COTCOT code available on the web. Sections 2 and 3 are the main contribution of the paper. In Section 4 , we illustrate these results with some nonacademic applications in aerospace control: the minimal time problem of orbit transfer, and the attitude control of a rigid body.

\section{SECOND ORDER OPTIMALITy CONDITIONS}

\subsection{General Lagrange problem}

The material of this section if taken from [5] (see also [3]).

Let $\left(X,\|\cdot\|_{X}\right)$ be a Banach space, densely imbedded into a Hilbert space $\left(H,\|\cdot\|_{H}\right)$. Let $C: X \rightarrow \mathbb{R}$, and $E: X \rightarrow \mathbb{R}^{n}$, with $n \geq 1$, be mappings of class $C^{2}$. We consider the minimization problem with equality constraint

$$
\min _{E(x)=0} C(x)
$$

\footnotetext{
${ }^{1}$ In practice, $N$ is often equal to $\mathbb{R}^{m}$. However, in the orbit transfer problem investigated in this article, we need to consider $N=S^{2}$, the unit sphere of $\mathbb{R}^{3}$, and $U=N$. Of course, the framework of this article applies when replacing $N$ by a chart.
} 
According to the Lagrange multipliers rule, if $x \in X$ is optimal then there exists a nontrivial couple $\left(\psi, \psi^{0}\right) \in$ $\mathbb{R}^{n} \times \mathbb{R}$ so that $\psi \cdot \mathrm{d} E(x)+\psi^{0} \mathrm{~d} C(x)=0$, where $\mathrm{d} E(x)$ (resp. $\left.\mathrm{d} C(x)\right)$ denotes the Fréchet derivative of $E$ (resp. $C$ ) at the point $x$. In other words, the point $x$ is a singular point of the mapping

$$
\begin{aligned}
\tilde{E}: X & \rightarrow \mathbb{R}^{n} \times \mathbb{R} \\
y & \mapsto(E(y), C(y)),
\end{aligned}
$$

and $\tilde{\psi} \cdot \mathrm{d} \tilde{E}(x)=0$, where $\tilde{\psi}=\left(\psi, \psi^{0}\right)$. The corank of $x$ is defined as the codimension of $\operatorname{Im~} \mathrm{d} \tilde{E}(x)$.

This is a first order necessary condition. The next result from [5] (see also [4]) provides second order optimality conditions. Recall that the negative index of a quadratic form $q$ is defined as the maximal dimension of subspaces $L$ on which $q$ is negative definite.

Theorem $1.1[5]$.

- If $x$ is a local minimum in $X$ of the problem (5), of corank $m$, then there exists a nontrivial pair of Lagrange multipliers $\tilde{\psi}=\left(\psi, \psi^{0}\right) \in \mathbb{R}^{n} \times \mathbb{R}$ so that the negative index of the quadratic form $\tilde{\psi} \cdot \mathrm{d}^{2} \tilde{E}(x)$ restricted to $\operatorname{ker} \mathrm{d} E(x)$ is less than or equal to $m-1$.

- Let $x \in X$ be a singular point of $\tilde{E}$, for a given nontrivial pair of Lagrange multipliers $\tilde{\psi}=\left(\psi, \psi^{0}\right) \in$ $\mathbb{R}^{n} \times \mathbb{R}$. Assume that there holds, for every $h \in X$,

$$
E(x+h)=E(x)+\mathrm{d} E(x) \cdot h+o\left(\|h\|_{H}\right), \quad \tilde{\psi} \cdot \tilde{E}(x+h)=\tilde{\psi} \cdot \tilde{E}(x)+\frac{1}{2} \tilde{\psi} \cdot \mathrm{d}^{2} \tilde{E}(x) \cdot(h, h)+o\left(\|h\|_{H}^{2}\right),
$$

as $\|h\|_{X}$ tends to zero. If moreover the quadratic form $\tilde{\psi} \cdot \mathrm{d}^{2} \tilde{E}(x)$ admits a continuous extension on $H$ that is $H$ positive definite on $\operatorname{ker} \mathrm{d} E(x)$, i.e. there exists a positive real number $\gamma$ so that $\tilde{\psi} \cdot \mathrm{d}^{2} \tilde{E}(x) \cdot(h, h) \geq$ $\gamma\|h\|_{H}^{2}$, for every $h \in \operatorname{ker} \mathrm{d} E(x)$, then $x$ is a local minimum in $X$ of the problem (5).

Remark 1.2. In the corank one case, there exists, up to a multiplying scalar, a unique nontrivial pair of Lagrange multipliers $\tilde{\psi}=\left(\psi, \psi^{0}\right)$. The quadratic form $Q$, defined as the restriction of $\tilde{\psi} \cdot \mathrm{d}^{2} \tilde{E}(x)$ to ker $\mathrm{d} E(x)$, is called intrinsic second order derivative of $F$ at $x$. The previous theorem mainly asserts that, if $Q$ is $H$ positive definite, then $x$ is a local minimum; conversely, if $x$ is a local minimum, then $Q$ is nonnegative. The aim of the conjugate time theory, presented further, is to try to compute the time at which this form ceases to be positive definite.

For singular points of corank greater than one, the situation is more intricate, and one has to deal with a pencil of quadratic forms and the notion of Morse index. If one of these quadratic forms is positive definite, then the trajectory is locally optimal in $L^{\infty}$ topology. However, conversely, the fact that each quadratic form of the pencil is indefinite is not sufficient to ensure that the trajectory is not optimal. The condition on the index is indeed stronger.

\subsection{Application to optimal control}

We now apply the previous results in the framework of optimal control theory, in order to derive necessary and/or sufficient second order optimality conditions. Consider the optimal control problem for the system (1), together with the cost (2), with the boundary conditions $M_{0}$ and $M_{1}$, the final time being fixed or not.

Definition 1.3. Let $T>0$. The end-point mapping of the system (1) is the mapping

$$
\begin{aligned}
E: M \times \mathbb{R}^{+} \times \mathcal{U} & \longrightarrow M \\
\left(x_{0}, T, u\right) & \longmapsto x\left(x_{0}, T, u\right),
\end{aligned}
$$

where $t \mapsto x\left(x_{0}, t, u\right)$ is the trajectory solution of $(1)$, associated to the control $u$, such that $x\left(x_{0}, 0, u\right)=x_{0}$.

If $\mathcal{U}$ is endowed with the $L^{\infty}$ topology, then this mapping is smooth.

Let $x(\cdot)$ be a trajectory of the system (1), associated to a smooth control $u$, on $[0, T]$. Then, the control $u$ can be extended smoothly to $[0, T+\varepsilon]$, where $\varepsilon>0$ is fixed. 
Definition 1.4.

- If the final time is fixed, $x(\cdot)$ is said to be locally optimal in $L^{\infty}$ topology (resp. locally optimal in $C^{0}$ topology), if it is optimal in a neighborhood of $u$ in $L^{\infty}$ topology (resp. in a neighborhood of $x(\cdot)$ in $C^{0}$ topology).

- If the final time is not fixed, $x(\cdot)$ is said to be locally optimal in $L^{\infty}$ topology if, for every neighborhood $V$ of $u$ in $L^{\infty}([0, T+\varepsilon], U)$, for every real number $\eta$ so that $|\eta| \leq \varepsilon$, for every control $v \in V$ satisfying $E\left(x_{0}, T+\eta, v\right)=E\left(x_{0}, T, u\right)$, there holds $C(T+\eta, v) \geq C(T, u)$.

Moreover, $x(\cdot)$ is said to be locally optimal in $C^{0}$ topology if, for every neighborhood $W$ of the trajectory $x(\cdot)$ in $M$, for every real number $\eta$ so that $|\eta| \leq \varepsilon$, for every trajectory $y(\cdot)$, associated to a control $v$ on $[0, T+\eta]$, contained in $W$, and satisfying $y(0)=x_{0}, y(T+\eta)=x(T)$, there holds $C(T+\eta, v) \geq C(T, u)$.

Remark 1.5. If $x(\cdot)$ is optimal (that is, globally optimal), then it is locally optimal in $C^{0}$ topology, and if so, then it is locally optimal in $L^{\infty}$ topology. The $C^{0}$ local optimality (resp. $L^{\infty}$ local optimality) is sometimes called strong local optimality (resp. weak local optimality).

The final time of our optimal control problem being fixed or not, the problem reduces to a minimization problem of the type (5). In this context, the results of the previous section can be immediately applied in order to derive second order conditions of optimality, in terms of positivity of the intrinsic second order derivative of the end-point mapping.

Note that, if the final time is not fixed, then the time variable has to be taken into account in the Hessian.

These conditions are however abstract, and in the next section we provide Legendre type conditions, ensuring the positivity of this second order derivative, and thus, leading to optimality results.

\subsection{Legendre type conditions}

The following theorem is standard (see $[3,5,7,10,19-21,25,28]$ ).

\section{Theorem 1.6.}

(1) If a trajectory $x(\cdot)$, associated to a control $u$, is optimal on $\left[0, t_{f}\right]$ in $L^{\infty}$ topology, then the Legendre condition holds along every extremal lift $\left(x(\cdot), p(\cdot), p^{0}, u(\cdot)\right)$ of $x(\cdot)$, that is

$$
\frac{\partial^{2} H}{\partial u^{2}}\left(x(\cdot), p(\cdot), p^{0}, u(\cdot)\right) \cdot(v, v) \leq 0, \quad \forall v \in \mathbb{R}^{m}
$$

(2) If the strong Legendre condition holds along the extremal $\left(x(\cdot), p(\cdot), p^{0}, u(\cdot)\right)$, that is, there exists $\alpha>0$ such that

$$
\frac{\partial^{2} H}{\partial u^{2}}\left(x(\cdot), p(\cdot), p^{0}, u(\cdot)\right) .(v, v) \leq-\alpha\|v\|^{2}, \quad \forall v \in \mathbb{R}^{m},
$$

then there exists $\varepsilon>0$ so that the trajectory $x(\cdot)$ is locally optimal in $L^{\infty}$ topology on $[0, \varepsilon]$. If the extremal is moreover normal, i.e. $p^{0} \neq 0$, then $x(\cdot)$ is locally optimal in $C^{0}$ topology on $[0, \varepsilon]$.

Remark 1.7. The Legendre condition, claimed in the first point of the theorem as a necessary condition for optimality, can be derived directly from the maximization condition in the maximum principle.

In the second point of the theorem, the strong Legendre condition actually ensures the $L^{2}$ positive definiteness of the intrinsic second order derivative of the end-point mapping on small intervals (see [5]). Note that the Banach space $L^{\infty}(0, T)$ is densely imbedded in the Hilbert space $L^{2}(0, T)$. This leads, according to the results of the previous section, to $L^{\infty}$ local optimality results only. However, under the strong Legendre condition, the maximized Hamiltonian of the maximum principle is smooth, and the theory of extremal fields leads to stronger results whenever the extremal is normal, namely local optimality in $C^{0}$ topology (see the references cited previously). 
We say that we are in the regular case whenever the strong Legendre condition holds along the extremal.

The previous theorem is not relevant in the case where $\frac{\partial^{2} H}{\partial u^{2}}=0$, for instance in the case of the time optimal problem for control affine systems. We introduce the following definition of [3].

Definition 1.8. An extremal $\left(x(\cdot), p(\cdot), p^{0}, u(\cdot)\right)$ is said totally singular whenever $\frac{\partial^{2} H}{\partial u^{2}}\left(x(\cdot), p(\cdot), p^{0}, u(\cdot)\right)=0$.

Theorem 1.9 [3].

(1) If a trajectory $x(\cdot)$, associated to a piecewise smooth control $u$, and having a totally singular extremal lift $\left(x(\cdot), p(\cdot), p^{0}, u(\cdot)\right)$, is optimal on $\left[0, t_{f}\right]$ in $L^{\infty}$ topology, then the Goh condition holds along the extremal, that is

$$
\left\{\frac{\partial H}{\partial u_{i}}, \frac{\partial H}{\partial u_{j}}\right\}=0
$$

along the extremal, where $\{\cdot, \cdot\}$ denotes the Poisson bracket on $T^{*} M$. Moreover, one has the generalized Legendre condition

$$
\left\{\left\{H, \frac{\partial H}{\partial u} \cdot v\right\}, \frac{\partial H}{\partial u} \cdot v\right\}+\left\{\frac{\partial^{2} H}{\partial u^{2}} \cdot(\dot{u}, v), \frac{\partial H}{\partial u} \cdot v\right\} \leq 0
$$

along the extremal, for every $v \in \mathbb{R}^{m}$.

(2) If the Goh condition holds along the extremal $\left(x(\cdot), p(\cdot), p^{0}, u(\cdot)\right)$, if the strong generalized Legendre condition holds along the extremal, that is, there exists $\alpha>0$ such that

$$
\left\{\left\{H, \frac{\partial H}{\partial u} \cdot v\right\}, \frac{\partial H}{\partial u} \cdot v\right\}+\left\{\frac{\partial^{2} H}{\partial u^{2}} \cdot(\dot{u}, v), \frac{\partial H}{\partial u} \cdot v\right\} \leq-\alpha\|v\|^{2},
$$

along the extremal, for every $v \in \mathbb{R}^{m}$, and if moreover the mapping $\frac{\partial f}{\partial u}\left(x_{0}, u(0)\right): \mathbb{R}^{m} \rightarrow T_{x_{0}} M$ is one-to-one, then there exists $\varepsilon>0$ so that the trajectory $x(\cdot)$ is locally optimal in $L^{\infty}$ topology on $[0, \varepsilon]$.

Remark 1.10. The Goh condition, which is a necessary condition for optimality, was first established in [18]. It follows from the finiteness of the index of the intrinsic second order derivative of the end-point mapping. Another necessary condition is the generalized Legendre condition, stated in $[1,22,23]$ and generalized in [3].

In the second point of the theorem, the strong Legendre condition actually ensures the $H^{-1}$ positive definiteness of the intrinsic second order derivative of the end-point mapping on small intervals (see [5]). Here, the notation $H^{-1}(0, T)$ stands for the Sobolev space defined as the dual of the space $H^{1}(0, T)$ of absolutely continuous functions with square integrable derivative. Note that the Banach space $L^{\infty}(0, T)$ is densely imbedded in the Hilbert space $H^{-1}(0, T)$. This leads, according to the results of the previous section, to local optimality results in $L^{\infty}$ topology only. However, under the strong Legendre condition, the maximized Hamiltonian of the maximum principle is smooth, and the theory of extremal fields leads to stronger results, namely local optimality in $C^{0}$ topology (see the references cited previously).

Example 1.11. A typical and important example of a totally singular extremal is provided by the time optimal problem for a single-input affine control system on the manifold $M$, namely, $\dot{x}(t)=f_{0}(x(t))+u(t) f_{1}(x(t))$, where $u(t) \in \mathbb{R}$, and $f_{0}, f_{1}$ are smooth vector fields on $M$. This kind of system will be investigated in more details in the next section. Of course, in this case, one has $\partial^{2} H / \partial u^{2}=0$ everywhere, and the Hamiltonian writes $H\left(x, p, p^{0}, u\right)=\left\langle p, f_{0}(x)\right\rangle+u\left\langle p, f_{1}(x)\right\rangle$. The strong generalized Legendre condition (11) reduces to $\left\langle p(\cdot),\left[f_{1},\left[f_{0}, f_{1}\right]\right](x(\cdot))\right\rangle>0$, where $[$,$] denotes the Lie bracket of vector fields, and is usually called generalized$ Legendre condition. Finally, the condition of injectivity of the second point of the theorem is automatically satisfied provided $f_{1}\left(x_{0}\right) \neq 0$.

To conclude this section, let us observe that, for optimal controls of corank greater than one, we obtained, on the one part, necessary conditions in terms of index, and on the other part, sufficient conditions in terms of positive definiteness of the second derivative. 
In the corank one case, the pencil of quadratic forms associated to the end-point mapping reduces to a unique (up to scalar) quadratic form, namely, the intrinsic second order derivative of the end-point mapping. In this case, we get almost necessary and sufficient conditions for optimality. More precisely, if the control is optimal then the latter quadratic form is nonnegative, and conversely, if the quadratic form is positive definite then the control is optimal. These conditions of positivity are ensured by Legendre type conditions, at least on small intervals. The aim of the conjugate time theory, introduced in the next section, is to characterize the time at which this quadratic form ceases to be positive definite.

\subsection{Conjugate times in the corank one case}

Let $x(\cdot)$ be an optimal trajectory on $[0, T]$ of the optimal control problem (1), (2), associated to the control $u$. Assume that $u$ is of corank one. In particular, the trajectory admits a unique (up to a scalar) extremal lift $\left(x(\cdot), p(\cdot), p^{0}, u(\cdot)\right)$ on $[0, T]$, which may be normal $\left(p^{0} \neq 0\right)$ or abnormal $\left(p^{0}=0\right)$. Assume further that the strong Legendre condition holds along the extremal, or, whenever the extremal is totally singular, assume that the strong generalized Legendre condition holds along the extremal, on $[0, T]$.

For every $t \in[0, T]$, denote by $Q_{t}$ the intrinsic second order derivative of the end-point mapping on $[0, t]$. Its expression depends on whether the final time is fixed or not in the optimal control problem (see Sect. 1.2). Then $Q_{t}$ is positive definite, for every $t \in[0, \varepsilon]$, where $\varepsilon>0$ is small enough.

Definition 1.12. Define the first conjugate time $t_{c}$ along the extremal $\left(x(\cdot), p(\cdot), p^{0}, u(\cdot)\right)$ as the supremum of times $t$ so that $Q_{t}$ is positive definite.

From the definition of a first conjugate time, it is clear that the trajectory $x(\cdot)$ is locally optimal in $L^{\infty}$ topology on $\left[0, t_{c}\right)$. It is however not clear that $x(\cdot)$ loses its optimality beyond $t_{c}$. For that fact to be true, one needs the quadratic form $Q_{t}$ to be indefinite for $t>t_{c}$. It may however happen, in degenerate cases, that $Q_{t}$ be nonnegative on an interval $\left[t_{c}, t_{c}+\eta\right]$, with $\eta>0$.

To avoid this kind of pathological behavior, one needs a further assumption. Of course, in an analytic framework this phenomenon does not occur, but we prefer to deal with less regularity assumptions. The following assumption, called strong regularity assumption, is standard in the calculus of variations.

(S) The control $u$ is of corank one on every subinterval $\left[t_{1}, t_{2}\right]$ of $[0, T]$.

Under this additional assumption, the quadratic form $Q_{t}$ is indeed indefinite on $[0, t]$, for $t>t_{c}$.

We summarize all results in the following theorem (see for instance [3]).

Theorem 1.13. Let $\left(x(\cdot), p(\cdot), p^{0}, u(\cdot)\right)$ be an extremal of corank one of the optimal control problem (1), (2), on $[0, T]$. Let $t_{c}$ denote the first conjugate time along this extremal.

- If the strong Legendre condition holds along the extremal, or, whenever the extremal is totally singular, if the strong generalized Legendre condition holds along the extremal, then the trajectory $x(\cdot)$ is locally optimal in $L^{\infty}$ topology on $\left[0, t_{c}\right)$.

If the extremal is moreover normal, and satisfies the strong Legendre condition, then $x(\cdot)$ is locally optimal in $C^{0}$ topology on $\left[0, t_{c}\right)$.

- Under the additional strong regularity assumption $(\mathrm{S})$, the extremal is not locally optimal in $L^{\infty}$ topology on $[0, t]$, for every $t>t_{c}$.

The aim is now to provide efficient algorithms to compute conjugate times. This will be achieved in the next section through the theory of Lagrangian manifolds. We will define the concept of geometric conjugate time, related to the exponential mapping, and show that it coincides with the concept of conjugate time.

In the traditional terminology of the calculus of variations, this exponential mapping approach is directly related to the theory of extremal fields. 


\section{Geometric conjugate time theory}

\subsection{Lagrangian manifolds, Hamiltonian vector fields}

Let $M$ be a smooth manifold of dimension $n$. Let $T^{*} M$ denote the cotangent bundle of $M, \pi: T^{*} M \rightarrow M$ the canonical projection, and $\omega$ the canonical symplectic form on $T^{*} M$. Recall that $\left(T^{*} M, \omega\right)$ is a symplectic manifold, that is, $\omega$ is a smooth 2 -form on $T^{*} M$ that is closed and nondegenerate.

Definition 2.1. A regular submanifold $L$ of $T^{*} M$ is said to be isotropic if its tangent space is isotropic at every point, i.e. the restriction of $\omega(z)$ to $T_{z} L \times T_{z} L$ is zero, for every $z \in L$. If moreover the dimension of $L$ is equal to $n$, then $L$ is said to be a Lagrangian submanifold of $T^{*} M$.

Definition 2.2. A diffeomorphism $f$ on $T^{*} M$ is said to be symplectic whenever $\varphi^{*} \omega=\omega$, where $\varphi^{*} \omega(z)$. $(u, v)=$ $\omega(\varphi(z)) \cdot(\mathrm{d} \varphi(z) \cdot u, \mathrm{~d} \varphi(z) \cdot v)$, for every $z \in T^{*} M$ and all $u, v \in T_{z}\left(T^{*} M\right)$.

Lemma 2.3. Let $L$ denote a Lagrangian submanifold of $N$, and $\varphi$ a symplectic diffeomorphism on L. Then $\varphi(L)$ is a Lagrangian submanifold of $N$.

Definition 2.4. For a smooth function $H$ on $T^{*} M$, denote by $\vec{H}$ the Hamiltonian vector field on $T^{*} M$ defined by $i_{\vec{H}} \omega=-\mathrm{d} H$, where $i_{\vec{H}} \omega(z) \cdot u \omega(z)(\vec{H}(z), u)$, for every $z \in T^{*} M$ and every $u \in T_{z}\left({ }^{*} M\right)$.

The vector field $\vec{H}$ is said to be Hamiltonian, and $H$ is called the Hamiltonian function.

Let $\varphi_{t}=\exp _{t}(\vec{H})$ denote the local one-parameter group on $T^{*} M$ which is the flow of the vector field $\vec{H}$. Lemma 2.3 implies that $L_{t}=\varphi_{t}(L)$ is a Lagrangian submanifold of $T^{*} M$.

Definition 2.5. Let $L$ be a Lagrangian submanifold of $T^{*} M$, and $z \in L$. A vector $z \in T_{z} L \backslash\{0\}$ is said to be vertical if $\mathrm{d} \pi(z) \cdot v=0$. The caustic is defined as the set of points $z \in L$ at which there exists a vertical vector.

Remark 2.6. Let $x_{0} \in M$; the fiber $L_{0}=T_{x_{0}}^{*} M$ is a Lagrangian submanifold of $T^{*} M$ whose vectors are all vertical. More generally, let $M_{0}$ be a regular submanifold of $M$; then, the set $M_{0}^{\perp}$ of elements $(x, p)$ of $T^{*} M$ so that $x \in M_{0}$ and $p \perp T_{x_{0}} M$ is a Lagrangian submanifold of $T^{*} M$.

\subsection{Jacobi equation, geometric conjugate times}

Definition 2.7. Let $\vec{H}$ be a Hamiltonian vector field on $T^{*} M$, and let $z(t)$ be a trajectory of $\vec{H}$ defined on $[0, T]$, i.e., $\dot{z}(t)=\vec{H}(z(t))$, for every $t \in[0, T]$. The differential equation on $[0, T]$

$$
\dot{\delta z}(t)=\mathrm{d} \vec{H}(z(t)) \delta z(t)
$$

is called Jacobi equation along $z(\cdot)$, or variational system along $z(\cdot)$.

A Jacobi field $J(t)$ is a nontrivial solution of the Jacobi equation along $z(\cdot)$. It is said to be vertical at the time $t$ whenever $\mathrm{d} \pi(z(t)) . J(t)=0$. In local coordinates, set $J(t)=(\delta x(t), \delta p(t))$; then, $J(\cdot)$ is vertical at the time $t$ whenever $\delta x(t)=0$.

A time $t_{c}$ is said to be geometrically conjugate if there exists a Jacobi field that is vertical at times 0 and $t_{c}$; the point $x\left(t_{c}\right)=\pi\left(z\left(t_{c}\right)\right)$ is then said to be geometrically conjugate to $x(0)=J(z(0))$.

Definition 2.8. For every $z_{0} \in T^{*} M$, let $z\left(t, z_{0}\right)$ denote the trajectory of $\vec{H}$ such that $z\left(0, z_{0}\right)=z_{0}$. The exponential mapping is defined by

$$
\exp _{t}\left(z_{0}\right)=\pi\left(z\left(t, z_{0}\right)\right)
$$

In local coordinates, denoting $z_{0}=\left(x_{0}, p_{0}\right) \in T^{*} M, z\left(t, x_{0}, p_{0}\right)=\left(x\left(t, x_{0}, p_{0}\right), p\left(t, x_{0}, p_{0}\right)\right)$, one has $\exp _{x_{0}, t}\left(p_{0}\right)=$ $x\left(t, x_{0}, p_{0}\right)$.

The following result, stated in local coordinates, easily follows from a geometric interpretation from the Jacobi equation. 
Proposition 2.9. Let $x_{0} \in M, L_{0}=T_{x_{0}}^{*} M$, and $L_{t}=\exp _{t}(\vec{H})\left(L_{0}\right)$. Then $L_{t}$ is a Lagrangian submanifold of $T^{*} M$, whose tangent space is spanned by Jacobi fields starting from $L_{0}$. Moreover, $x\left(t_{c}\right)$ is geometrically conjugate to $x_{0}$ if and only if the mapping $\exp _{x_{0}, t_{c}}$ is not immersive at $p_{0}$.

Remark 2.10. The notion of geometric conjugate point can be generalized as follows. Let $M_{1}$ be a regular submanifold of $M$, and $M_{1}^{\perp}=\left\{(x, p) \mid x \in M_{1}, p \perp T_{x} M_{1}\right\}$. The time $T$ is said focal, and $q(T)$ is said a focal point, if there exists a Jacobi field $J(t)=(\delta x(t), \delta p(t))$ such that $\delta x(0)=0$ and $J(T)$ is tangent to $M_{1}^{\perp}$.

The aim of next section is mainly to provide conditions under which a geometric conjugate time is a first conjugate time. As before, we distinguish between the regular case and the totally singular case.

\subsection{The regular case}

Consider the optimal control problem for the system (1) with the cost $(2)$. Let $\left(x(\cdot), p(\cdot), p^{0}, u(\cdot)\right)$ be a reference extremal. In this section we make the following assumptions.

(L) The strong Legendre condition holds along the extremal, that is, there exists $\alpha>0$ such that

$$
\frac{\partial^{2} H}{\partial u^{2}}\left(x(\cdot), p(\cdot), p^{0}, u(\cdot)\right) \cdot(v, v) \leq-\alpha\|v\|^{2}, \quad \forall v \in \mathbb{R}^{m} .
$$

(S) The control $u$ is of corank one on every subinterval (assumption of strong regularity).

The Implicit Function Theorem, and the maximization condition (4) of the Pontryagin maximum principle, imply that extremal controls can be computed as smooth functions $u_{r}(t)=u_{r}(x(t), p(t))$, in a neighborhood of $u$. It is then possible to define, locally, the so-called reduced Hamiltonian

$$
H_{r}(x, p)=H\left(x, p, u_{r}(x, p)\right) .
$$

Then, every extremal satisfies $\dot{x}=\frac{\partial H_{r}}{\partial p}(x, p), \dot{p}=-\frac{\partial H_{r}}{\partial x}(x, p)$, or, denoting $z=(x, p)$,

$$
\dot{z}(t)=\vec{H}_{r}(z(t))
$$

where $\vec{H}_{r}$ denotes the Hamiltonian vector field associated to the Hamiltonian function $H_{r}$.

Remark 2.11. Note that the control $u_{r}(x, p)$ is homogeneous in $p$ of degree 0 , i.e., $u_{r}(x, \lambda p)=u_{r}(x, p)$, and the solutions of the reduced system are such that $x\left(t, x_{0}, \lambda p_{0}\right)=x\left(t, x_{0}, p_{0}\right), p\left(t, x_{0}, \lambda p_{0}\right)=\lambda p\left(t, x_{0}, p_{0}\right)$, for every real number $\lambda$.

In this context, the exponential mapping writes $\exp _{x_{0}, t}\left(p_{0}\right)=x\left(t, x_{0}, p_{0}\right)$, where $\left(x\left(t, x_{0}, p_{0}\right), p\left(t, x_{0}, p_{0}\right)\right)$ is the solution of (13) starting from $\left(x_{0}, p_{0}\right)$ at $t=0$.

The following fundamental result relates the conjugate time theory to the optimality status (see $[5,11,25])$.

Theorem 2.12. Under assumptions $(\mathrm{L})$ and $(\mathrm{S})$, the first geometric conjugate time coincides with the first conjugate time along $\left(x(\cdot), p(\cdot), p^{0}, u(\cdot)\right)$, denoted by $t_{c}$.

Therefore, the trajectory $x(\cdot)$ is locally optimal on $\left[0, t_{c}\right)$ in $L^{\infty}$ topology (in $C^{0}$ topology whenever the extremal is normal); if $t>t_{c}$, then the trajectory $x(\cdot)$ is not locally optimal in $L^{\infty}$ topology on $[0, t]$.

The domain of the exponential mapping depends, on the one part, on whether the final time $t_{f}$ is fixed or not, and on the other part, on whether the extremal is normal or abnormal. Hence, the test for conjugate times is different in each of the following cases.

(1) Normal case. In the normal case, one has $p^{0}<0$, and since the initial adjoint vector $\left(p(0), p^{0}\right)$ is defined up to a multiplicative scalar, one can normalize it so that $p^{0}=-1$. 
(a) Final time fixed. If the final time is fixed, the domain of $\exp _{x_{0}, t}$ is a subset of $T_{x_{0}}^{*} M$, that is locally diffeomorphic to $\mathbb{R}^{n}$. In this case, the method to compute conjugate times is the following. One has to compute numerically the Jacobi fields $J_{i}(t)=\left(\delta x_{i}(t), \delta p_{i}(t)\right), i=1, \ldots, n$, corresponding to the initial conditions $\delta x_{i}(0)=0$ and $\delta p_{i}(0)=e_{i}, i=1, \ldots, n$, where $\left(e_{i}\right)_{1 \leq i \leq n}$ represents the canonical basis of $\mathbb{R}^{n}$, and to compute

$$
\operatorname{rank}\left(\delta x_{1}(t), \ldots, \delta x_{n}(t)\right)
$$

this rank being equal to $n$ outside a conjugate time, and being lower than or equal to $n-1$ at a conjugate time.

(b) Final time not fixed. In this case, the Pontryagin maximum principle yields the additional condition $H=0$ along the extremal. One has to take into account this condition when computing the Jacobi fields. We thus introduce the set

$$
X=\left\{p_{0} \in T_{x_{0}}^{*} M \mid H_{r}\left(x_{0}, p_{0}\right)=0\right\}
$$

It is a submanifold of $M$ of codimension one provided $\frac{\partial H_{r}}{\partial p}\left(x_{0}, p_{0}\right)=f\left(x_{0}, u\left(x_{0}, p_{0}\right)\right) \neq 0$. Then, the domain of $\exp _{x_{0}, t}$ is a subset of $X$, that is locally diffeomorphic to $\mathbb{R}^{n-1}$.

In this case, in order to compute conjugate times, we provide three equivalent tests.

- Test 1. Consider the $(n-1)$ dimensional vector space of Jacobi fields $J_{i}(t)=\left(\delta x_{i}(t), \delta p_{i}(t)\right)$, $i=1, \ldots, n-1$, vertical at 0 , satisfying $\delta p_{i}(0) \in T_{p(0)} X$, that is, satisfying

$$
f\left(x_{0}, u\left(x_{0}, p_{0}\right)\right) . \delta p_{i}(0)=0 .
$$

One has to compute numerically these Jacobi fields, and to determine at what time

$$
\operatorname{rank} \mathrm{d} \pi\left(J_{1}(t), \ldots, J_{n-1}(t)\right)=\operatorname{rank}\left(\delta x_{1}(t), \ldots, \delta x_{n-1}(t)\right) \leq n-2 .
$$

- Test 2. Another possibility is to compute numerically the Jacobi fields $J_{i}(t)=\left(\delta x_{i}(t), \delta p_{i}(t)\right)$, $i=1, \ldots, n$, corresponding to the initial conditions $\delta p_{i}(0)=e_{i}, i=1, \ldots, n$, where $\left(e_{i}\right)_{1 \leq i \leq n}$ represents the canonical basis of $\mathbb{R}^{n}$, and to compute

$$
\operatorname{rank}\left(\delta x_{1}(t), \ldots, \delta x_{n}(t)\right)
$$

this rank being equal to $n-1$ outside a conjugate time, and being lower than or equal to $n-2$ at a conjugate time.

- Test 3. Note the derivative of the exponential mapping with respect to $t$ is equal to the dynamics $f$ of the system. Hence, it is also possible to consider a basis $\left(\delta p_{1}(0), \ldots, \delta p_{n-1}(0)\right)$ satisfying (14), to compute numerically the corresponding Jacobi fields $J_{i}(t)=\left(\delta x_{i}(t), \delta p_{i}(t)\right)$, $i=1, \ldots, n-1$, and to determine a zero of

$$
\operatorname{det}\left(\delta x_{1}(t), \ldots, \delta x_{n-1}(t), f(x(t), u(x(t), p(t)))\right) \text {. }
$$

Indeed, by assumption the Hamiltonian is not equal to zero along the extremal, and thus $\dot{x}(t)$ is transverse to $\mathrm{d} \pi\left(J_{1}(t), \ldots, J_{n-1}(t)\right)$.

(2) Abnormal case. In the abnormal case, one has $p^{0}=0$, and since the initial adjoint vector $\left(p(0), p^{0}\right)$ is defined up to a multiplicative scalar, one can normalize it so that $\|p(0)\|=1$. In other words, $p(0) \in S^{n-1}$, where $S^{n-1}$ denotes the sphere centered at 0 with radius 1 in $T_{x_{0}}^{*} M$. 
(a) Final time fixed. If the final time is fixed, the domain of $\exp _{x_{0}, t}$ is a subset of $S^{n-1}$, that is locally diffeomorphic to $\mathbb{R}^{n-1}$. In this case, we compute numerically the Jacobi fields $J_{i}(t)=$ $\left(\delta x_{i}(t), \delta p_{i}(t)\right), i=1, \ldots, n-1$, corresponding to the initial conditions $\delta x_{i}(0)=0$ and $\delta p_{i}(0) \in$ $T_{p_{0}} S^{n-1}$, that is, $p_{0} . \delta p_{i}(0)=0$, for $i=1, \ldots, n-1$. We then compute

$$
\operatorname{rank}\left(\delta x_{1}(t), \ldots, \delta x_{n-1}(t)\right),
$$

this rank being equal to $n-1$ outside a conjugate time, and being lower than or equal to $n-2$ at a conjugate time.

(b) Final time not fixed. In this case, the Pontryagin maximum principle yields the additional condition $H=0$ along the extremal. As previously, we thus introduce the set

$$
X=\left\{p_{0} \in T_{x_{0}}^{*} M \mid\left\|p_{0}\right\|=1, H_{r}\left(x_{0}, p_{0}\right)=0\right\} .
$$

From the condition $H_{r}\left(x_{0}, p_{0}\right)=\left\langle p_{0}, f\left(x_{0}, u\left(x_{0}, p_{0}\right)\right)\right\rangle=0$, we easily prove that $X$ is a submanifold of $M$ of codimension two, provided $\frac{\partial H_{r}}{\partial p}\left(x_{0}, p_{0}\right)=f\left(x_{0}, u\left(x_{0}, p_{0}\right)\right) \neq 0$.

Then, the domain of $\exp _{x_{0}, t}$ is a subset of $X$, that is locally diffeomorphic to $\mathbb{R}^{n-2}$.

To compute conjugate times, we proceed as follows. Consider the $(n-2)$ dimensional vector space of Jacobi fields $J_{i}(t)=\left(\delta x_{i}(t), \delta p_{i}(t)\right), i=1, \ldots, n-2$, vertical at 0 , satisfying $\delta p_{i}(0) \in T_{p(0)} X$, that is, satisfying $f\left(x_{0}, u\left(x_{0}, p_{0}\right)\right) \cdot \delta p_{i}(0)=0$, and $p_{0} . \delta p_{i}(0)=0$. We then determine at what time

$$
\operatorname{rank} \mathrm{d} \pi\left(J_{1}(t), \ldots, J_{n-2}(t)\right)=\operatorname{rank}\left(\delta x_{1}(t), \ldots, \delta x_{n-2}(t)\right) \leq n-3 .
$$

Remark 2.13. The minimal time problem is an important particular case, that corresponds to $f^{0}=1$, and where the final time is not fixed. The Hamiltonian writes $H=\langle p, f(x, u)\rangle+p^{0}$, and one has to distinguish between the normal case $\left(p^{0}<0\right)$ and the abnormal case $\left(p^{0}=0\right)$.

Example 2.14 (academic example). Consider the minimal time problem for the control system in $\mathbb{R}^{2}$

$$
\dot{x}_{1}(t)=u(t), \quad \dot{x}_{2}(t)=1-u(t)^{2}+x_{1}(t)^{2},
$$

and the initial condition $x_{1}(0)=x_{2}(0)=0$. The Hamiltonian is $H=p_{1} u+p_{2}\left(1-u^{2}+x_{1}^{2}\right)+p^{0}$, and the extremal control writes $u=p_{1} / 2 p_{2}$. One computes easily $\exp _{t}(\lambda)=\left(\lambda \sin t, t-\lambda^{2} \frac{\sin 2 t}{2}\right)$, and, using this explicit formulation, one gets that the first conjugate for the trajectory $\left(x_{1}(t)=0, x_{2}(t)=1\right)$, associated to the control $u=0$, is $t_{c}=\pi$. The associated extremal is normal. A numerical simulation permits to check this result. Figure 1 represents $\operatorname{det}\left(\delta x_{1}(t), \delta x_{2}(t), f\right)$, where $f$ is the dynamics of the system, that indeed vanishes at $t=\pi$.

Another example (the sub-Riemannian case). Consider the optimal control problem

$$
\dot{x}=\sum_{i=1}^{m} u_{i} f_{i}(x), \min _{u} \int_{0}^{T}\left(\sum_{i=1}^{m} u_{i}^{2}\right)^{1 / 2} \mathrm{~d} t,
$$

where the final time $T$ is fixed. The cost represents the length of a curve tangent to the distribution $D=$ $\operatorname{Vect}\left(f_{1}, \ldots, f_{m}\right)$, the fields $f_{i}$ being orthonormal. This cost does not depend on the parametrization of the curve, and applying Maupertuis principle, the problem amounts to minimizing the energy $\int_{0}^{T} \sum_{i=1}^{m} u_{i}^{2} \mathrm{~d} t$. Finally, for this particular case, one has $f(x, u)=\sum_{i=1}^{m} u_{i} f_{i}(x)$, and $f^{0}(x, u)=\sum_{i=1}^{m} u_{i}^{2}$. In the normal case, one can set $p^{0}=-1 / 2$, and the maximization condition leads to $u_{i}=\left\langle p, f_{i}(x)\right\rangle$. The reduced Hamiltonian writes

$$
H_{r}=\frac{1}{2} \sum_{i=1}^{m} u_{i}^{2}=\frac{1}{2} \sum_{i=1}^{m}\left\langle p, f_{i}(x)\right\rangle^{2} .
$$




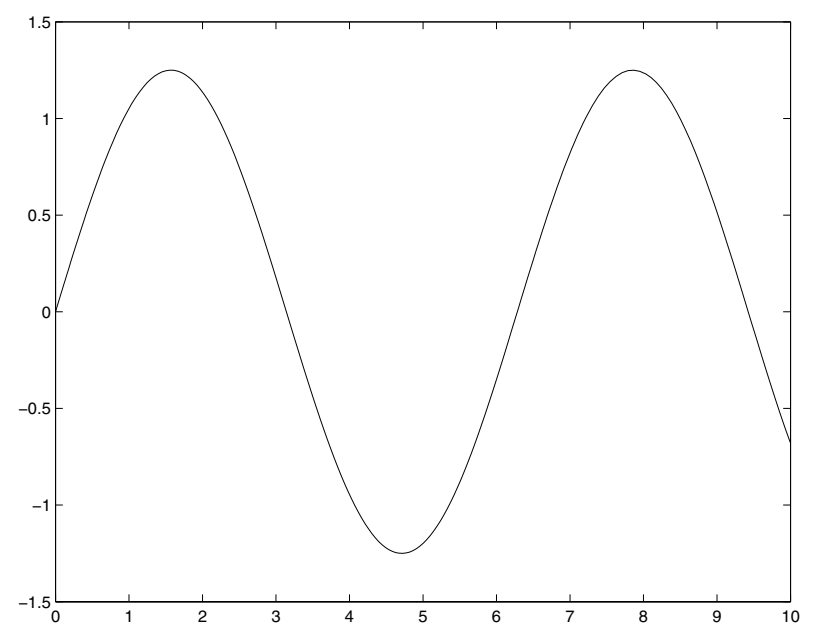

Figure 1. $\operatorname{det}\left(\delta x_{1}(t), \delta x_{2}(t), f\right)$.

One can normalize the trajectories on the level set $H_{r}=1 / 2$. Extremals are solutions of

$$
\dot{x}=\frac{\partial H_{r}}{\partial p}, \dot{p}=-\frac{\partial H_{r}}{\partial x} .
$$

The previously described algorithm applies. Denote by $J_{i}(t)=\left(\delta x_{i}(t), \delta p_{i}(t)\right)$ the Jacobi field so that $\delta x_{i}(0)=0$ et $\delta p_{i}(0)=e_{i}$, where $\left(e_{i}\right)_{1 \leq i \leq n}$ is the canonical basis of $\mathbb{R}^{n}$. A conjugate time corresponds to vanishing the determinant $D(t)=\operatorname{det}\left(\delta x_{1}(t), \ldots, \delta x_{n}(t)\right)$.

It is possible to reduce the computation, noticing that extremal solutions of (15) satisfy $x\left(t, x_{0}, \lambda p_{0}\right)=$ $x\left(\lambda t, x_{0}, p_{0}\right), p\left(t, x_{0}, \lambda p_{0}\right)=\lambda p\left(\lambda t, x_{0}, p_{0}\right)$. Hence, one of the Jacobi fields is trivial. More precisely, considering the variation $\alpha(\varepsilon)=\left(x_{0},(1+\varepsilon) p_{0}\right)$, if $J(t)$ denotes the associated Jacobi field, then $\mathrm{d} \pi(J(t))$ is collinear to $\dot{x}(t)$.

The algorithm then reduces to test the rank of $\left(\delta x_{1}(t), \ldots, \delta x_{n-1}(t)\right)$, where $J_{i}(t)=\left(\delta x_{i}(t), \delta p_{i}(t)\right)$ is the Jacobi field such that $\delta x_{i}(0)=0$, and $\delta p_{i}(0) \perp p_{0}$.

Fixing $H_{r}=1 / 2$, the domain of the exponential mapping is the cylinder $\mathbb{R} \times S^{m-1} \times \mathbb{R}^{n-m}$. The time $t_{c}$ is conjugate if and only if the mapping $\exp _{x_{0}, t_{c}}$ is not immersive at $\left(t_{c}, p_{0}\right)$.

Recall that an extremal $z(t)=(x(t), p(t))$ solution of $(15)$ is said to be strict on $[0, T]$ if the trajectory $x(\cdot)$ admits only one extremal lift, up to a scalar. Then, for such an extremal, the trajectory $x(\cdot)$ is locally $C^{0}$ optimal up to the first conjugate time.

Example 2.15. Consider the so-called Martinet case, with $n=3, m=2$, and $f_{1}=\frac{\partial}{\partial x}+\frac{y^{2}}{2} \frac{\partial}{\partial z}, f_{2}=\frac{\partial}{\partial y}$. Simulations are lead along the extremal starting from the point

$$
x(0)=y(0)=z(0)=0, p_{x}(0)=-0.9, p_{y}(0)=0.19, p_{z}(0)=100 .
$$

In Figure 2 are drawn, one the one part, the projection on the plane $(x y)$ of the associated trajectory (it is an Euler elastica), on the other part, the determinant of Jacobi fields, that vanishes at about $t_{c}=1.112$.

\subsection{Discussion in the totally singular case}

The situation in the totally singular case is far more intricate than in the regular case. In Theorem 1.9, we saw that, if a trajectory $x(\cdot)$ of system (1) is optimal for the cost (2), and admits a totally singular extremal lift $\left(x(\cdot), p(\cdot), p^{0}, u(\cdot)\right)$, then the Goh condition (9) must hold along this extremal. 

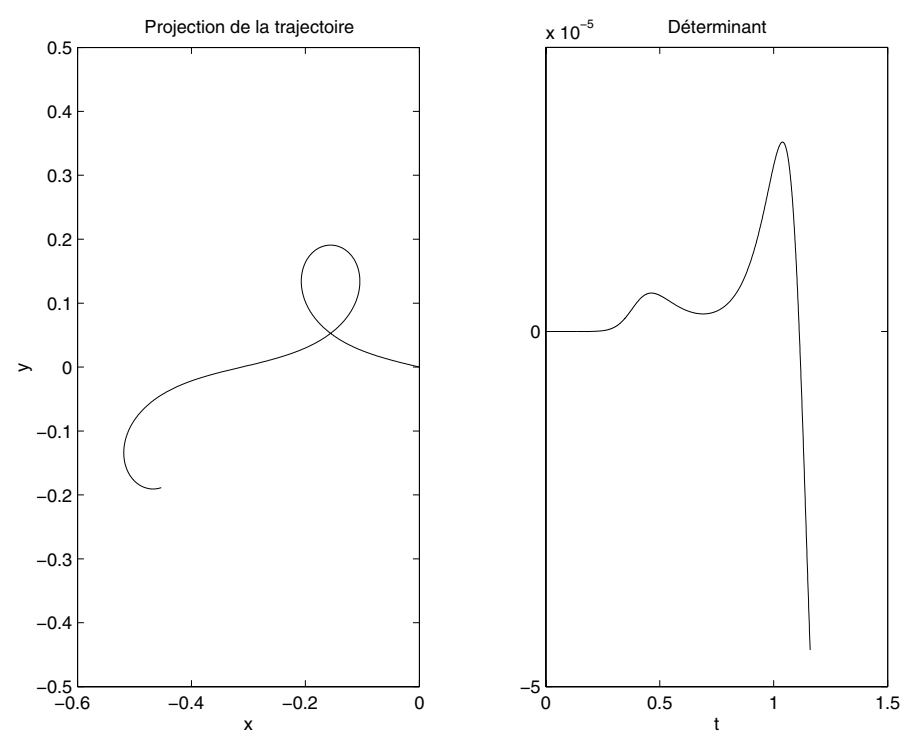

Figure 2. Determinant in the Martinet case.

This condition is empty whenever the control is scalar, that is, $u(t) \in \mathbb{R}$. It imposes however a strong restriction along the extremal for multi-input control systems. For instance, if one deals with multi-input affine control systems

$$
\dot{x}(t)=f_{0}(x(t))+\sum_{i=1}^{m} u_{i}(t) f_{i}(x(t))
$$

with $m \geq 2$, one can prove that successive differentiations of the Goh condition with respect to $t$ yield an infinite number of independent relations (see [15] for details). As a consequence, generic multi-input affine control systems do not admit time optimal trajectories. On the opposite, in nongeneric situations, one is unable in general to derive the extremal controls as dynamic feedback functions $u(x(t), p(t))$.

It is thus relevant to restrict our study to single-input affine systems, i.e., $m=1$. Indeed, generic such systems do admit time optimal trajectories, and under generic assumptions one is able to compute extremal controls $u(x(t), p(t))$, and thus to define a concept of exponential mapping.

Note that the situation of sub-Riemannian systems of rank two, $\dot{x}(t)=u_{1}(t) f_{1}(x(t))+u_{2}(t) f_{2}(x(t))$, is completely similar and can be actually reduced to the previous case.

Remark 2.16. Using the so-called integral transformation (see next section), it is actually possible to reduce a multi-input affine system of the form (16) to a nonlinear system on a manifold of smaller dimension, provided that the vector fields $f_{i}, i=1, \ldots, m$, mutually commute. This situation is nongeneric but is important in classical mechanics.

\subsection{The minimal time case of single-input control affine systems}

In this section, we consider the minimal time problem for the single-input affine system

$$
\dot{x}(t)=f_{0}(x(t))+u(t) f_{1}(x(t))
$$

where $f_{0}$ and $f_{1}$ are smooth vector fields on the $n$-dimensional manifold $M$, and $u(t) \in \mathbb{R}$. Every minimal time trajectory $x(\cdot)$ is singular, that is, its associated control $u(\cdot)$ is a singularity of the end-point mapping.

Let $x(\cdot)$ be such a reference minimal time trajectory. We make the following assumptions.

$\left(\mathbf{H}_{\mathbf{0}}\right)$ The trajectory $x(\cdot)$ is smooth and injective. 
For the sake of simplicity, we assume $u=0$.

$\left(\mathbf{H}_{\mathbf{1}}\right)$ The set $\left\{a \mathrm{~d}^{k} f_{0} \cdot f_{1}(x(t)), k \in \mathbb{N}\right\}$ has codimension one.

This assumption implies in particular that the singularity of the end-point mapping is of codimension one (i.e., $x(\cdot)$ is of corank one), or, equivalently, that the first Pontryagin cone $K(t)=\operatorname{Im} \mathrm{d} E_{t}(u)$ is equal to the subspace of codimension one Span $\left\{a \mathrm{~d}^{k} f_{0} . f_{1}(x(t)), k \in \mathbb{N}\right\}$, where $a \mathrm{~d} f_{0} \cdot f_{1}=\left[f_{0}, f_{1}\right]$.

Then, the trajectory $x(\cdot)$ admits a unique (up to scalar) extremal lift $\left(x(\cdot), p(\cdot), p^{0}, u(\cdot)\right)$, which may be normal $\left(p^{0}<0\right)$ or abnormal $\left(p^{0}=0\right)$. The adjoint vector $p(\cdot)$ is actually oriented by using the condition $p^{0} \leq 0$ of the maximum principle.

The following assumption is, up to the sign, the generalized Legendre condition. Notice that, due to the particular form of the control system (17), the strong generalized Legendre condition (11), up to the sign, reduces to that condition.

$\left(\mathbf{H}_{2}\right) a \mathrm{~d}^{2} f_{1} \cdot f_{0}(x(t)) \notin K(t)$ along the trajectory.

Introduce the Hamiltonian lifts of the vector fields $f_{0}$ and $f_{1}$, namely, $h_{0}(x, p)=\left\langle p, f_{0}(x)\right\rangle, h_{1}(x, p)=\left\langle p, f_{1}(x)\right\rangle$. It follows from the maximum principle that, along the extremal lift of $x(\cdot)$, there must hold $h_{1}(x(\cdot), p(\cdot))=0$. Derivating with respect to $t$, one gets $\left\langle p(\cdot),\left[f_{0}, f_{1}\right](x(\cdot)\rangle\left\{h_{0}, h_{1}\right\}(x(\cdot), p(\cdot))=0\right.$, where $\{$, $\}$ denotes the Poisson bracket. A further derivation leads to $\left\{h_{0},\left\{h_{0}, h_{1}\right\}\right\}(x(\cdot), p(\cdot))+u(\cdot)\left\{h_{1},\left\{h_{0}, h_{1}\right\}\right\}(x(\cdot), p(\cdot))=0$. Therefore, under the previous assumptions, extremals are associated to the controls

$$
u(x, p)=\frac{\left\{h_{0},\left\{h_{1}, h_{0}\right\}\right\}(x, p)}{\left\{h_{1},\left\{h_{0}, h_{1}\right\}\right\}(x, p)},
$$

satisfy the constraints $h_{1}=\left\{h_{0}, h_{1}\right\}=0$, and are solutions of

$$
\dot{x}=\frac{\partial \hat{H}}{\partial p}, \dot{p}=-\frac{\partial \hat{H}}{\partial x},
$$

where $\hat{H}$ is the reduced Hamiltonian, $\hat{H}(x, p)=h_{0}(x, p)+u(x, p) h_{1}(x, p)$.

Then, the exponential mapping is defined as follows. For every $x_{0} \in M$ and every $p_{0} \in T_{x_{0}}^{*} M$, satisfying $h_{1}\left(x_{0}, p_{0}\right)=\left\{h_{0}, h_{1}\right\}\left(x_{0}, p_{0}\right)=0$, set $\exp _{x_{0}, t}\left(p_{0}\right)=x\left(t, x_{0}, p_{0}\right)$, where $\left(x\left(\cdot, x_{0}, p_{0}\right), p\left(\cdot, x_{0}, p_{0}\right)\right)$ is the extremal solution of $(18)$, so that $x\left(0, x_{0}, p_{0}\right)=x_{0}$ and $p\left(0, x_{0}, p_{0}\right)=p_{0}$.

We recall the terminology used in [11].

\section{Definition 2.17.}

(1) If $h_{0}>0$ :

(a) if $\left\{\left\{h_{1}, h_{0}\right\}, h_{1}\right\}>0$, the trajectory is said to be hyperbolic;

(b) if $\left\{\left\{h_{1}, h_{0}\right\}, h_{1}\right\}<0$, the trajectory is said to be elliptic.

(2) If $h_{0}=0$, the trajectory is said to be exceptional.

Actually, the trajectory $x(\cdot)$ is elliptic or hyperbolic if and only if its extremal lift $x(\cdot), p(\cdot), p^{0}, u(\cdot)$ is normal (i.e. $p^{0}<0$ ), and it is exceptional if and only if its extremal lift is abnormal $\left(\right.$ i.e. $\left.p^{0}=0\right)$.

Notice that, due to the generalized Legendre condition (particular case of the strong generalized Legendre condition (11)), only hyperbolic and exceptional trajectories may be time minimal. Actually, elliptic trajectories are candidates for time maximality.

The domain of $\exp _{x_{0}, t}$ depends on the nature of the trajectory.

- If the trajectory $x(\cdot)$ is hyperbolic or elliptic, then we are in the normal case. Previously, in the regular case, we normalized the initial adjoint vector $\left(p(0), p^{0}\right)$ so that $p^{0}=-1$, and took into account the condition $H=0$ given by the maximum principle. Here, for the minimal time problem, since $f^{0}=1$, it is more judicious to normalize $\left(p(0), p^{0}\right)$ so that $\|p(0)\|=1$; then the corresponding associated Lagrange multiplier $p^{0}$ is computed using the condition $H=0$, and $p^{0}=-\left\langle p(0), f\left(x_{0}, u\left(x_{0}, p(0)\right)\right)\right\rangle$. With this 
normalization, the domain of $\exp _{x_{0}, t}$ is an open subset of

$$
\left\{p_{0} \in T_{x_{0}}^{*} M \mid\left\|p_{0}\right\|=1, h_{1}\left(x_{0}, p_{0}\right)=\left\{h_{0}, h_{1}\right\}\left(x_{0}, p_{0}\right)=0\right\} .
$$

- If the trajectory $x(\cdot)$ is exceptional, then we are in the abnormal case, and up to scalar we assume that $p(0)=p_{0} \in S^{n-1}$, where $S^{n-1}$ is the unit sphere centered at 0 in $T_{x_{0}}^{*} M$. Then, the domain of $\exp _{x_{0}, t}$ is an open subset of

$$
\left\{p_{0} \in T_{x_{0}}^{*} M \mid\left\|p_{0}\right\|=1, h_{0}\left(x_{0}, p_{0}\right)=h_{1}\left(x_{0}, p_{0}\right)=\left\{h_{0}, h_{1}\right\}\left(x_{0}, p_{0}\right)=0\right\}
$$

Integral transformation. We need a further assumption.

$\left(\mathbf{H}_{3}\right)$ The vector field $f_{1}$ is transverse to the reference trajectory $x(\cdot)$.

In a tubular neighborhood of $x(\cdot)$, the field $f_{1}$ is identified to $f_{1}=\frac{\partial}{\partial x_{n}}$. Then, locally, the system writes

$$
\dot{\tilde{x}}=f\left(\tilde{x}, x_{n}\right), \quad \dot{x}_{n}=g\left(\tilde{x}, x_{n}\right)+u,
$$

where $\tilde{x}=\left(x_{1}, \ldots, x_{n-1}\right)$.

Definition 2.18. The integral transformation consists in considering as a new control the control $v=x_{n}$. Consider then the reduced system (19), written as $\dot{\tilde{x}}=f(\tilde{x}, v)$.

The Hamiltonian of this system is $\tilde{H}(\tilde{x}, \tilde{p}, v)=\langle\tilde{p}, f(\tilde{x}, v)\rangle$.

Lemma 2.19. The triple $(x, p, u)$ is an extremal of the system (17) if and only if $\left(\tilde{x}, \tilde{p}, x_{n}\right)$ is an extremal of the reduced system (19). Moreover,

$$
\frac{\partial}{\partial t} \frac{\partial H}{\partial u}-\frac{\partial \tilde{H}}{\partial x_{n}}, \quad \frac{\partial}{\partial u} \frac{\partial^{2}}{\partial t^{2}} \frac{\partial H}{\partial u}-\frac{\partial^{2} \tilde{H}}{\partial x_{n}^{2}}
$$

In particular, the strong Legendre condition for the reduced system is equivalent to the generalized Legendre condition for the initial affine system.

In order to derive some normal forms in a tubular neighborhood of $x(\cdot)$, we further need the following technical assumption.

$\left(\mathbf{H}_{4}\right)$ For every $t \in[0, T]$, the vectors $a \mathrm{~d}^{k} f_{0} \cdot f_{1}(x(t)), k=0, \ldots, n-2$, are linearly independent along the reference trajectory. In particular, $K(t)=\operatorname{Span}\left\{a \mathrm{~d}^{k} f_{0} \cdot f_{1}(x(t)) \mid k=0, \ldots, n-2\right\}$.

In the exceptional case where $f_{0}(x(t)) \in K(t)$, we assume moreover that

- if $n=2$, then $f_{0}(x(t))$ and $f_{1}(x(t))$ are independent, for every $t \in[0, T]$;

- if $n \geq 3$, then $f_{0}(x(t)) \notin \operatorname{Span}\left\{a \mathrm{~d}^{k} f_{0} \cdot f_{1}(x(t)), k=0, \ldots, n-3\right\}$.

Under these assumptions, according to [11], it is possible to derive, up to feedback equivalence, normal forms of the system in a tubular neighborhood of $x(\cdot)$, which permit to express the intrinsic second order derivative, represented by an explicit differential operator.

Recall that two affine control systems $\dot{x}=f_{0}(x)+u f_{1}(x)$ and $\dot{y}=f_{0}^{\prime}(y)+v f_{1}^{\prime}(y)$ are called feedback equivalent if there exists a smooth diffeomorphism $\Phi:(x, u) \mapsto(y, v)$, of the form $y=\varphi(x), v=\alpha(x)+\beta(x) u$, such that $\mathrm{d} \Phi(x) \cdot\left(f_{0}(x)+u f_{1}(x)\right)=f_{0}^{\prime}(y)+v f_{1}^{\prime}(y)$. Note that singular trajectories are invariant under feedback equivalence.

We distinguish between the elliptic/hyperbolic case, and the exceptional case. 


\section{Elliptic and hyperbolic cases.}

Lemma 2.20 [11]. Suppose that $x(\cdot)$ is elliptic or hyperbolic, and $n \geq 2$. Then the system (17) is in a $C^{0}$ neighborhood of $x(\cdot)$ feedback equivalent to

$$
f_{0}=\frac{\partial}{\partial x_{1}}+\sum_{i=2}^{n-1} x_{i+1} \frac{\partial}{\partial x_{i}}+\sum_{i, j=2}^{n} a_{i j}\left(x_{1}\right) x_{i} x_{j} \frac{\partial}{\partial x_{1}}+\sum_{i=1}^{n} Z_{i} \frac{\partial}{\partial x_{i}}, \quad f_{1}=\frac{\partial}{\partial x_{n}},
$$

where $a_{n, n}(t)<0$ on $[0, T]$ and the 1-jet (resp. the 2-jet) of $Z_{i}, i=2, \ldots, n$ (resp. $Z_{1}$ ) along $x(\cdot)$ is equal to zero.

The k-jet is defined in the following way. Let $V=\sum_{k=1}^{n} V_{k} \frac{\partial}{\partial x_{k}}$ a vector field. Since $x(\cdot)$ is given by $x_{1}(t)=t$ and $x_{i}(t)=0, i=2, \ldots, n$, the component $V_{k}$ can be written in a neighborhood of $x(\cdot)$ as $\sum_{p=1}^{+\infty} j_{p} V_{k}$, where $j_{0} V=V_{\mid x(\cdot)}$, and $j_{1} V_{k}=\sum_{i=2}^{n} a_{i}^{k}\left(x_{1}\right) x_{i}, j_{2} V_{k}=\sum_{i, j=2}^{n} b_{i j}^{k}\left(x_{1}\right) x_{i} x_{j}, \ldots$ Set $j_{i} V=\sum_{k=1}^{n} j_{i} V_{k} \frac{\partial}{\partial x_{k}}$. Then $\sum_{i=0}^{k} j_{i} V$ is called the $k$-jet of $V$ along $x(\cdot)$.

In these conditions, the controllable part of the system is $\left(x_{2}, \ldots, x_{n}\right)$, the singular reference trajectory is $x(t)=(t, 0, \ldots, 0)$, and the intrinsic second order derivative along $x(\cdot)$ is identified to $\int_{0}^{T} \sum_{i, j=2}^{n} a_{i j}(t) \xi_{i}(t) \xi_{j}(t) \mathrm{d} t$, where $\dot{\xi}_{2}=\xi_{3}, \ldots, \dot{\xi}_{n-1}=\xi_{n}, \dot{\xi}_{n}=v$. Set $y=\xi_{2}$. Then it can be written as $Q_{T \mid G}$, where $Q_{T}(y)=$ $\int_{0}^{T} q_{T}(y) \mathrm{d} t$ and $q_{T}(y)=\sum_{i, j=0}^{n-2} b_{i j} y^{(i)} y^{(j)}$, with $b_{i-2, j-2}=\frac{a_{i j}+a_{j i}}{2}$, and where $G$ is the following space corresponding to the kernel of the first derivative,

$$
G=\left\{y \mid y^{(2(n-2))} \in L^{2}(0, T), y^{(i)}(0)=y^{(i)}(T)=0, i=0, \ldots, n-2\right\} .
$$

Integrating by parts, we get the following result (see $[11,27])$.

Lemma 2.21. The quadratic form $Q_{T}$ is represented on $G$ by the operator $D_{T}$, that is, $Q_{T}(y)=\left(D_{T} y, y\right)_{L^{2}}$, where $(,)_{L^{2}}$ denotes the usual scalar product in $L^{2}(0, T)$, and the operator $D_{T}$ is

$$
D_{T}=\frac{1}{2} \sum_{i=0}^{n-2}(-1)^{i} \frac{\mathrm{d}^{i}}{\mathrm{~d} t^{i}} \frac{\partial q}{\partial y^{(i)}}=\sum_{i, j=0}^{n-2}(-1)^{j} \frac{\mathrm{d}^{j}}{\mathrm{~d} t^{j}} b_{i j} \frac{\mathrm{d}^{i}}{\mathrm{~d} t^{i}} .
$$

The spectrum of $D_{T}$ on $G$ is empty. Hence this space has to be enlarged, so that the spectrum is not trivial, and so that the representation Lemma 2.21 still holds. We thus set

$$
F=\left\{y \mid y^{(n-2)} \in L^{2}(0, T), y^{(i)}(0)=y^{(i)}(T)=0, i=0, \ldots, n-3\right\} .
$$

Then, $T$ is a conjugate time along $x(\cdot)$ whenever there exists $y \in F$ such that $y^{(2(n-2))} \in L^{2}(0, T)$ and $D_{T} y=0$.

Lemma 2.22. For every $f \in L^{2}(0, T)$, if $T$ is not a conjugate time, then there exists $y \in F$ unique such that $y^{(2(n-2))} \in L^{2}(0, T)$ and $D_{T} y=f$. Let $L$ denote the operator $f \mapsto y$, considered as an operator from $L^{2}(0, T)$ into $L^{2}(0, T)$; it is selfadjoint and compact.

Let $t_{c}$ be the first conjugate time of the operator $D$. The following result is due to [11].

Theorem 2.23. Under the previous assumptions, the reference singular trajectory $x(\cdot)$ is time minimizing in the hyperbolic case, and time maximizing in the elliptic case, on $\left[0, t_{c}\right)$, among all trajectories contained in a tubular neighborhood of $x(\cdot)$. Moreover, $x(\cdot)$ is not time extremal on $[0, t]$ in $L^{\infty}$ topology, for every $t>t_{c}$.

Remark 2.24. Under our assumptions, in dimension 2 , the operator $D$ is equal to $b_{0} I d$, and thus $t_{c}=+\infty$. 


\section{Exceptional case.}

Lemma 2.25 [11]. Suppose that $x(\cdot)$ is exceptional, and that $n \geq 3$. Then the system (17) is in a $C^{0}$-neighborhood of $x(\cdot)$ feedback equivalent to

$$
f_{0}=\frac{\partial}{\partial x_{1}}+\sum_{i=1}^{n-2} x_{i+1} \frac{\partial}{\partial x_{i}}+\sum_{i, j=2}^{n} a_{i j}\left(x_{1}\right) x_{i} x_{j} \frac{\partial}{\partial x_{n}}+\sum_{i=1}^{n-1} x_{n} f_{i}\left(x_{1}\right) \frac{\partial}{\partial x_{i}}+\sum_{i=1}^{n} Z_{i} \frac{\partial}{\partial x_{i}}, \quad f_{1}=\frac{\partial}{\partial x_{n-1}},
$$

where $a_{n-1, n-1}(t)>0$ on $[0, T]$, and the 1-jet (resp. 2-jet) of $Z_{i}, i=1, \ldots, n-1$ (resp. $Z_{n}$ ) along $\gamma$ is equal to 0 . Moreover the feedback is such that $\varphi$ is a germ of diffeomorphism along $x(\cdot)$ that satisfies

(i) $\varphi\left(x_{1}, 0, \ldots, 0\right)=\left(x_{1}, 0, \ldots, 0\right)$;

(ii) $\frac{\partial \varphi}{\partial x_{n-1}}=(0, \ldots, 0, *, 0)$,

and $\alpha, \beta$ are real functions defined in a neighborhood of $x(\cdot)$ such that $\beta$ does not vanish along $x(\cdot)$, and $\alpha_{\mid x(\cdot)}=0$.

Set $x_{1}=t+\xi$. The controllable part of the system is $\left(\xi, x_{2}, \ldots, x_{n-1}\right)$, the reference singular trajectory is $x(t)=(t, 0, \ldots, 0)$, and the intrinsic second order derivative along $x(\cdot)$ is identified to $\int_{0}^{T} \sum_{i, j=2}^{n-1} a_{i j}(t) \xi_{i}(t) \xi_{j}(t) \mathrm{d} t$, where $\dot{\xi}_{1}=\xi_{2}, \ldots, \dot{\xi}_{n-2}=\xi_{n-1}, \dot{\xi}_{n-1}=v$, and $\xi_{i}(0)=\xi_{i}(T)=0, i=1, \ldots, n-1$. It can be written into two different ways.

(1) If $\xi=x_{1}-t$, it can be written as $Q_{/ G}$, where $Q(\xi)=\int_{0}^{T} q(\xi) \mathrm{d} t$ and $q(\xi)=\sum_{i, j=1}^{n-2} b_{i j} \xi^{(i)} \xi^{(j)}$, with $b_{i-1, j-1}=\frac{a_{i j}+a_{j i}}{2}$, and where $G$ is the following space corresponding to the kernel of the first derivative,

$$
G=\left\{\xi \mid \xi^{(2(n-2))} \in L^{2}(0, T), \xi^{(i)}(0)=\xi^{(i)}(T)=0, i=0, \ldots, n-2\right\} .
$$

Let $D$ be the operator representing $Q$. There holds $Q(\xi)=(\xi, D \xi)_{L^{2}}$, where

$$
D=\frac{1}{2} \sum_{i=1}^{n-2}(-1)^{i} \frac{\mathrm{d}^{i}}{\mathrm{~d} t^{i}} \frac{\partial q}{\partial y^{(i)}}=\sum_{i, j=1}^{n-2}(-1)^{j} \frac{\mathrm{d}^{j}}{\mathrm{~d} t^{j}} b_{i j} \frac{\mathrm{d}^{i}}{\mathrm{~d} t^{i}}
$$

(2) It can be expressed in function of $x_{2}$ as the quadratic form $Q_{1 / G_{1}}$, where $Q_{1}\left(x_{2}\right)=\int_{0}^{T} q_{1}\left(x_{2}\right) \mathrm{d} t$, and $q_{1}\left(x_{2}\right)=\sum_{i, j=0}^{n-3} b_{i+1, j+1} x_{2}^{(i)} x_{2}^{(j)}$, and where $G_{1}$ corresponds to the kernel of the first derivative,

$$
G_{1}=\left\{x_{2} \mid x_{2}^{(2(n-3))} \in L^{2}(0, T), x_{2}^{(i)}(0)=x_{2}^{(i)}(T)=0, i=0, \ldots, n-3, \text { and } \int_{0}^{T} x_{2}(t) \mathrm{d} t=0\right\} .
$$

Let $D_{1}$ be the operator representing $Q_{1}$. There holds $Q_{1}\left(x_{2}\right)=\left(x_{2}, D_{1} x_{2}\right)_{L^{2}}$, where

$$
D_{1}=\frac{1}{2} \sum_{i=0}^{n-3}(-1)^{i} \frac{\mathrm{d}^{i}}{\mathrm{~d} t^{i}} \frac{\partial q}{\partial y^{(i)}}=\sum_{i, j=0}^{n-3}(-1)^{j} \frac{\mathrm{d}^{j}}{\mathrm{~d} t^{j}} b_{i+1, j+1} \frac{\mathrm{d}^{i}}{\mathrm{~d} t^{i}}
$$

Note that $Q(\xi)=Q_{1}(\dot{\xi})$ and $D=-\frac{\mathrm{d}}{\mathrm{d} t} D_{1} \frac{\mathrm{d}}{\mathrm{d} t}$. As previously, the spectral study of these operators has to be made on larger spaces,

- $F=\left\{\xi \mid \xi^{(n-2)} \in L^{2}(0, T), \xi^{(i)}(0)=\xi^{(i)}(T)=0, i=0, \ldots, n-3\right\}$ for the operator $D$;

- $F_{1}=\left\{x_{2} \mid x_{2}^{(n-3)} \in L^{2}(0, T), x_{2}^{(i)}(0)=x_{2}^{(i)}(T)=0, i=0, \ldots, n-4\right\}$ for $D_{1}$ if $n \geq 4$ (if $n=3$, no condition is imposed).

Lemma $2.26[11,27]$. Let $t_{c}$ (resp. $t_{c c}$ ) denote the first conjugate time of $Q$ on $F$ (resp. of $Q_{1}$ on $F_{1}$ ). There holds $0<t_{c c}<t_{c}$. 


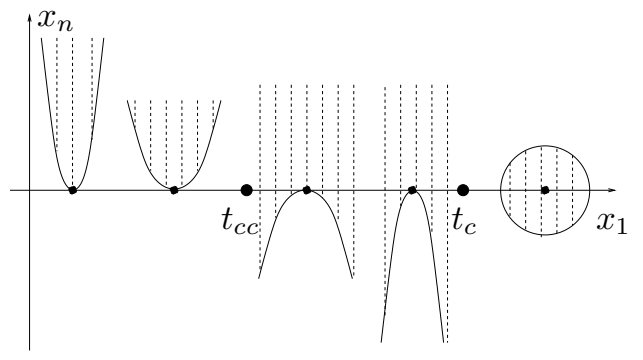

(a) Exceptional case

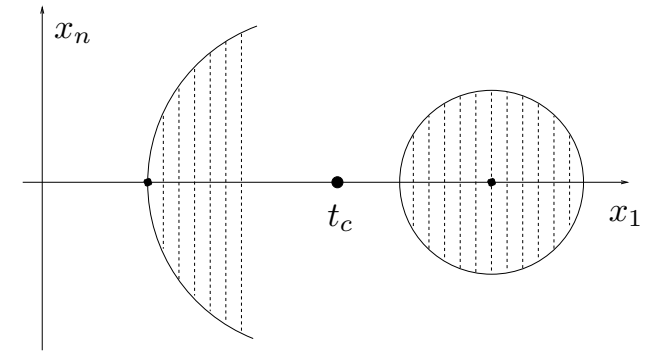

(b) Hyperbolic case

Figure 3. Shape of the accessible set around $x(t)$, in the plane $\left(x_{1}, x_{n}\right)$.

The following result is due to [11].

Theorem 2.27. Under the previous assumptions, in the exceptional case, the reference trajectory $x(\cdot)$ is time minimizing on $\left[0, t_{c c}\right)$, among all trajectories contained in a tubular neighborhood of $x(\cdot)$. It is not time minimizing on $[0, t]$ in $L^{\infty}$ topology, for every $t>t_{c c}$.

Remark 2.28. If $n=3$, then under our assumptions $t_{c c}=+\infty$.

Remark 2.29. In [27], a refined spectral analysis leads to a precise description of the asymptotics of the accessible set along the reference singular trajectory. The contact happens to be of order two under the previous assumptions. In the exceptional case, geometrically, the first conjugate time $t_{c c}$ corresponds to a change of concavity, in the $\left(x_{1}, x_{n}\right)$ plane, of the accessible set (responsible for the loss of time optimality), whereas the time $t_{c}$ corresponds to a topological bifurcation of the accessible set, that becomes locally open at $x(t)$, for $t>t_{c}$ (see Fig. 3). In the hyperbolic case, the time $t_{c c}$ does not exist.

We next provide some algorithms to compute conjugate times.

\section{Algorithms in the elliptic and hyperbolic cases.}

- Test 1. Using the integral transformation brings the system into the regular situation studied previously. Consider the reduced extremal system $\dot{\tilde{x}}=\frac{\partial \tilde{H}}{\partial \tilde{p}}, \dot{\tilde{p}}=-\frac{\partial \tilde{H}}{\partial \tilde{x}}$, and denote by $\tilde{J}_{1}(t), \ldots, \tilde{J}_{n-2}(t)$ the $n-2$ Jacobi fields, vertical at 0 , where $\delta \tilde{p}(0)$ satisfies $\langle\delta \tilde{p}(0), \tilde{p}(0)\rangle=0$. One then has to determine numerically at what time

$$
\operatorname{rank} \mathrm{d} \tilde{\pi}\left(\tilde{J}_{1}(t), \ldots, \tilde{J}_{n-2}(t)\right) \leq n-3 .
$$

Remark 2.30. The test only makes sense whenever $n \geq 3$. If $n=2$, then under the previous assumptions there is no conjugate time.

- Test 2. This second test is intrinsic and does not require the integral transformation. Consider the Jacobi fields solutions of the Jacobi equation associated to the initial system (17) with the linearized constraints $\mathrm{d} h_{1}=\mathrm{d}\left\{h_{0}, h_{1}\right\}=0$, with $\delta p(0)$ satisfying $\langle\delta p(0), p(0)\rangle=0$, and $\delta x(0) \in \mathbb{R} f_{1}(x(0))$. In other words, we consider a basis $\left(\delta x_{1}(0), \ldots, \delta x_{n}(0), \delta p_{1}(0), \ldots, \delta p_{n}(0)\right)$ such that

$$
\begin{aligned}
& \left\langle\delta p_{i}(0), p(0)\right\rangle=0, \\
& \left\langle\delta p_{i}(0), f_{1}(x(0))\right\rangle+\left\langle p_{i}(0), \mathrm{d} f_{1}(x(0)) . \delta x_{i}(0)\right\rangle=0, \\
& \left\langle\delta p_{i}(0),\left[f_{0}, f_{1}\right](x(0))\right\rangle+\left\langle p_{i}(0), \mathrm{d}\left[f_{0}, f_{1}\right](x(0)) . \delta x_{i}(0)\right\rangle=0, \\
& \delta x_{i}(0) \in \mathbb{R} f_{1}(x(0)),
\end{aligned}
$$

and we compute the $n-2$ associated Jacobi fields. We then compute numerically at what time

$$
\operatorname{rank}\left(\mathrm{d} \pi\left(J_{1}(t), \ldots, J_{n-2}(t)\right), f_{1}(x(t))\right)=\operatorname{rank}\left(\delta x_{1}(t), \ldots, \delta x_{n-2}(t), f_{1}(x(t))\right) \leq n-2 .
$$


Since the vector field $f_{0}$ is transverse to the Pontryagin cone along the trajectory, this is equivalent to test the vanishing of the determinant

$$
\operatorname{det}\left(\mathrm{d} \pi\left(J_{1}(t), \ldots, J_{n-2}(t)\right), f_{1}(x(t)), f_{0}(x(t))\right) .
$$

Algorithm in the exceptional case. Consider the restriction of extremals on the energy level $h_{0}=0$. We present a test without using the integral transformation (see [16] for details). Consider the $n-3$ Jacobi fields solutions of the Jacobi equation associated to the initial system (17) with linearized constraints $\mathrm{d} h_{1}=$ $\mathrm{d}\left\{h_{0}, h_{1}\right\}=\mathrm{d} h_{0}=0, \delta p(0)$ satisfying $\langle\delta p(0), p(0)\rangle=0$, and $\delta x(0) \in \mathbb{R} f_{1}(x(0))$, and we determine at what time

$$
\operatorname{rank}\left(\mathrm{d} \pi\left(J_{1}(t), \ldots, J_{n-3}(t)\right), f_{1}(x(t)), f_{0}(x(t))\right) \leq n-2 .
$$

Since the vector field $a \mathrm{~d}^{2} f_{1} \cdot f_{0}$ is transverse to the Pontryagin cone along the trajectory, this is equivalent to test the vanishing of the determinant

$$
\operatorname{det}\left(\mathrm{d} \pi\left(J_{1}(t), \ldots, J_{n-3}(t)\right), f_{1}(x(t)), f_{0}(x(t)), a \mathrm{~d}^{2} f_{1} \cdot f_{0}(x(t))\right) .
$$

Remark 2.31. The test only makes sense whenever $n \geq 4$. If $n=3$, then under the previous assumptions there is no conjugate time.

\section{The COTCOT code}

In this section, we introduce the COTCOT code, designed to compute extremals in the case of smooth Hamiltonian systems, and to obtain the associated conjugate points with respect to the index performance of the underlying optimal control problem. It can be downloaded at http://www.n7.fr/apo/cotcot (see the technical report [13]).

Below, we briefly explain how to use this routine, for the academic Example 2.14. Consider the minimum time control for the system

$$
\dot{x}_{1}(t)=u(t), \quad \dot{x}_{2}(t)=1-u(t)^{2}+x_{1}(t)^{2},
$$

where the extremities are fixed, $x(0)=x_{0}, x(T)=x_{1}$, and where $u(t) \in \mathbb{R}$. According to the maximum principle, the regular minimizing curves are the projection of extremals such that

$$
\dot{z}(t)=\vec{H}_{r}(z(t))
$$

where $H_{r}(z)=p_{1}^{2} / 4 p_{2}+p_{2}\left(1+x_{1}^{2}\right)-1$ is the smooth regular Hamiltonian defined on the open subset $\Sigma=$ $\left\{p_{2} \neq 0\right\}$, and where $\vec{H}_{r}=\left(\partial H_{r} / \partial p,-\partial H_{r} / \partial x\right)$. Since we have boundary conditions, the extremals we are interested in are $B C$-extremals. They are zeros of the shooting mapping defined by

$$
S:\left(t_{f}, p_{0}\right) \mapsto \Pi\left(\exp _{t_{f}}\left(x_{0}, p_{0}\right)\right)-x_{f}
$$

where $\exp _{t}\left(z_{0}\right)=z\left(t, z_{0}\right)$ is the solution of $(24)$ for the initial condition $z_{0}, \Pi:(x, p) \mapsto x$ is the canonical projection, and $p_{0} \in \mathbb{R}^{n}$ is such that $H_{r}\left(x_{0}, p_{0}\right)=0$, since the final time $t_{f}$ is free. The (local) optimality of such extremals is checked by a rank test on the subspaces spanned by the Jacobi fields along the trajectory. These fields are solutions of the variational equation $\delta \dot{z}(t)=\mathrm{d} \vec{H}_{r}(z(t)) \delta z(t)$, with suitable initial conditions. The aim of the code COTCOT (which stands for Conditions of Order Two, COnjugate Times) is to provide the numerical tools

1. to integrate smooth Hamiltonian systems such as (24);

2. to solve the associated shooting equation defined by (25);

3. to compute the corresponding Jacobi fields along the extremal;

4. to evaluate the resulting conjugate points, if any. 

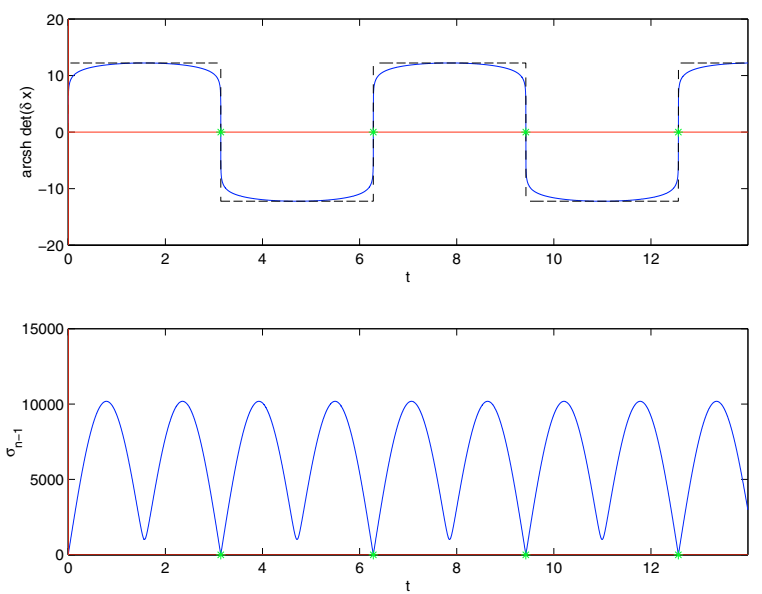

FiguRE 4. Result of the command main.

The package is intended for a standard Unix system with Matlab (version 6 or higher), Adifor (version 2.0 or higher), and a Fortran compiler known as $\mathbf{f 7 7}$. The automatic differentiation software Adifor, which is required (see [6]), can be downloaded at www-unix.mcs.anl.gov/autodiff/ADIFOR. The COTCOT archive can be downloaded at the URL www.n7.fr/apo/cotcot. The Fortran code defining the Hamiltonian is automatically differentiated twice and MEX-files for Matlab are generated.

The installation procedure is performed in three steps. First, the user should retrieve and uncompress the COTCOT archive. Then, from the parent directory cotcot/, the user should run the command make, so as to generate the code and compile it. Finally, from the folder main/, the user should launch Matlab, and try the command main. Among other printing, the user should get Figure 4 as well as the following final result:

The computation performed is analyzed next. We proceed in five steps.

Defining the Hamiltonian. The only user provided code is the Fortran subroutine defining the Hamiltonian $H$ of the system (24), stored in the file, the signature of which is

SUBROUTINE HFUN(T, N, Z, LPAR, PAR, H)

Obviously, the Hamiltonian may be time dependent. Moreover, additional parameters may be used (see Rem. 3.3 below). In our specific example, the code essentially amounts to:

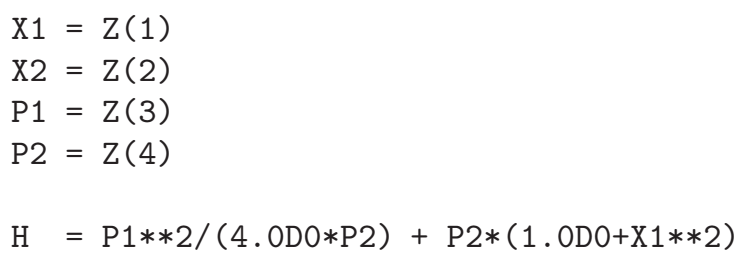

Note that, for the sake of robustness, dimensions are checked (MEXERRMSGTXT calls). Then, going back to the parent directory and running the command make, the Hamiltonian equation (24) and the variational system are generated by automatic differentiation, and compiled to produce MEX-files callable from Matlab.

Remark 3.1. The dimension $n$ must be lower or equal to the half of the constant N2MAX (maximum value of $2 n$ ) defined in include/constants.h. An error during the Matlab run is generated otherwise. In this case, the value of the constant has to be updated properly, and the code has to be generated again. 
Computing extremals. Going to the matlab/ subfolder, and launching Matlab, the mapping $\exp _{t}$ is computed by exphvfun:

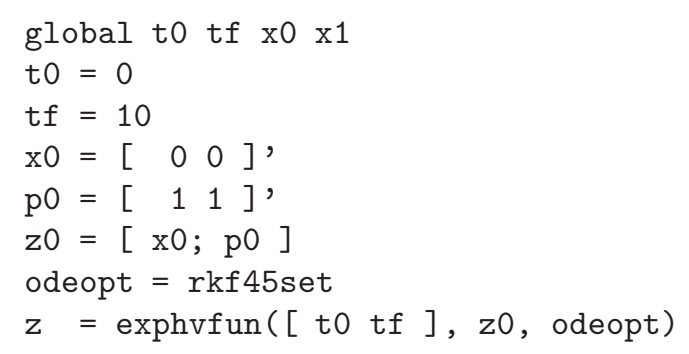

Remark 3.2. The underlying algorithm is Netlib Runge-Kutta one-step ODE integrator RKF45 (see [26]), whose parameters are managed with $r k f 45 g e t$ and $r k f 45$ set, and then passed to exphvfun.

Computing BC-extremals. The final time $t_{f}$ being free, we seek extremals in the level set $\left\{H_{r}=0\right\}$. As a result, the shooting mapping $(25)$ is evaluated by $S\left(t_{f}, p_{0}\right)=\left(\Pi\left(\exp _{t_{f}}\left(x_{0}, p_{0}\right)\right)-x_{f}, H_{r}\left(x_{0}, p_{0}\right)\right)$. The Hamiltonian is computed by exphvfun:

$\mathrm{h}=\mathrm{hfun}(\mathrm{t} 0, \mathrm{z} 0)$

Accordingly, denoting $\xi=\left(t_{f}, p_{0}\right)$, the shooting function is defined by (see main/sfun.m):

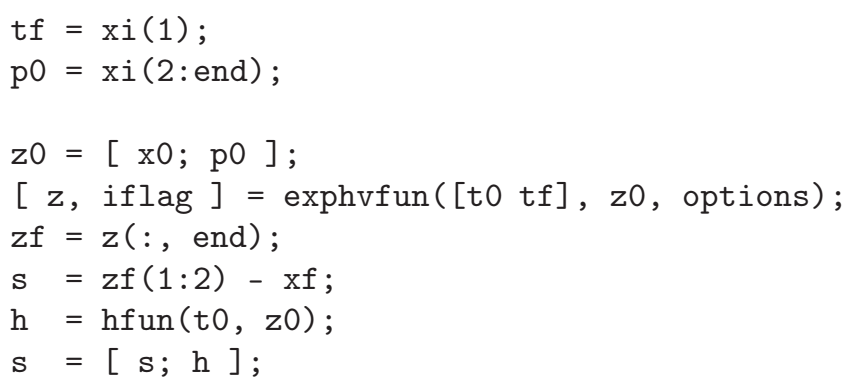

Remark 3.3. Any number of additional parameters can be passed to hfun. All of them must be real, or real matrices. They are vectorized to form one row vector which is the PAR argument of the Fortran subroutine HFUN. Furthermore, all Matlab commands provided in the package (exphvfun, expdhvfun...), accept such additional parameters that will be passed to the Hamiltonian.

\section{Typeset}

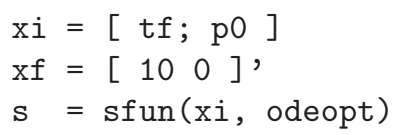

Zeros of the shooting mapping can be computed by any available solver, e.g., fsolve, or the faster and more robust function hybrd which is a Matlab port of Netlib HYBRD Newton solver provided with the COTCOT package. In our example, convergence is obtained with the initial guess $t f=10$ and $p_{0}=(1,1)$ :

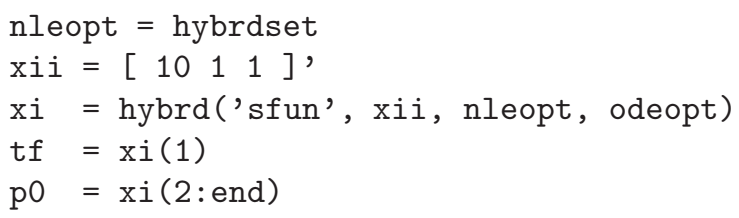

Remark 3.4. As for the ODE integrator (see Rem. 3.2), HYBRD parameters are managed with hybrdget and hybrdset. 
Computing Jacobi fields. The initial value of the Jacobi field has first to be defined, for instance, by

$[$ dummy, dpo ] $=\operatorname{gram}(\mathrm{p} 0)$

$\mathrm{dzO}=[0 ; 0 ; \mathrm{dpO}]$

so that $\delta p(0)$ belongs to the tangent space $T_{p(0)} \mathbf{S}^{n-1}$ (Gram-Schmidt orthonormalization, help gram). Since we must integrate the variational system along the previous extremal, the standard trick is to integrate both systems, Hamiltonian and variational, with the relevant initial conditions. Therefore, we extend the system and left-concatenate the extremal to the Jacobi field:

$\mathrm{z} 0=[\mathrm{x} 0 ; \mathrm{p} 0]$

$\mathrm{dzO}=[\mathrm{z} 0 \mathrm{dzO}]$

The sibling of exphvfun for the (extended) variational system is expdhvfun:

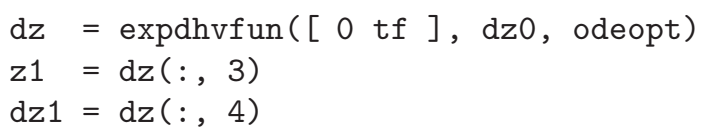

More generally, a full basis of Jacobi fields is computed in the same way by providing a matrix instead of a single vector. At each point, the image of the upper half matrix is the subspace whose rank must be tested for conjugate points. The command expdhvfun then returns the concatenation of these matrices (each of them extended by the extremal, concatenated to the left) at each point of the time array $t$ (standard vectorized input/output).

Computing conjugate points. The conjugate point test consists in checking a rank condition. To this end, a singular value decomposition is performed (see function main/draw.m). For minimum time problems, in the regular case it is equivalent to find a zero of the determinant of the projections of Jacobi fields on the $x$-space with the dynamics. For instance, the test at the final point is:

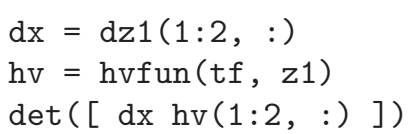

where hvfun computes $\vec{H}_{r}$. The function dfun evaluates this determinant at an arbitrary time $t$ for given initial conditions (see main/dfun.m). Hence, conjugate points are computed by finding its roots. As before, we use the hybrd solver and finally get (with an initial guess of 3.0 for $t_{c}$ ):

tci $=3.0 \quad \%$ initialization

tc $=$ hybrd('dfun', tci, nleopt, $0, \mathrm{dz} 0$, odeopt)

Indeed, the first conjugate time of our system is $t_{c, 1}=\pi$. The code main/main.m computes several conjugate points in this way.

Credits. The authors are grateful to Adifor $^{2}$ and Netlib ${ }^{3}$ people for making their codes available.

\section{Applications}

\subsection{Minimum time orbit transfer}

\section{Kepler equations}

The minimal time control of a satellite is considered [14]. In contrast with Section 4.2 where the rigid body itself is taken into account, the satellite is treated here as a single point in the Earth central field. The motion is driven by the controlled Kepler equations (we neglect second and higher order terms in the potential)

$$
\ddot{q}=-q \frac{\mu}{r^{3}}+\frac{F}{m}
$$

\footnotetext{
2 www-unix.mcs.anl.gov/autodiff/ADIFOR

3 netlib.enseeiht.fr
} 
TABle 1. Physical constants.

\begin{tabular}{ll}
\hline Variable & Value \\
\hline$\mu$ & $5165.8620912 \mathrm{Mm}^{3} \cdot \mathrm{h}^{-2}$ \\
$\beta$ & $1.42 e-2 \mathrm{Mm}^{-1} \cdot \mathrm{h}$ \\
$m_{0}$ & $1500 \mathrm{~kg}$ \\
\hline
\end{tabular}

where $r=|q|=\left(q_{1}+q_{2}+q_{3}\right)^{1 / 2}$ is the norm of the position vector $q$ in $\mathbb{R}^{3}$, where $F$ is the thrust of the engine, and where $m$ is the mass. The latter is besides varied to reflect the fuel consumption and one has $\dot{m}=-\beta|F|$. As before, $|\cdot|$ denotes the Euclidean norm in $\mathbb{R}^{3}$. A typical transfer consists in reaching the geostationnary orbit from a low eccentric one, the final mass of the satellite being unprescribed. The physical constants $\mu$ and $\beta$ involved in the dynamics are provided in Table 1 . We define the energy $E=1 / 2 \dot{q}^{2}-\mu / r$, the angular momentum $c=q \times \dot{q}$, and restrict the equation of motion (26) to the so-called elliptic domain [12], defined by $M=\left\{(q, \dot{q}) \in \mathbb{R}^{6} \mid E<0, c \neq 0\right\}$. The thrust $F$ is expressed in a moving frame. Several choices are available, among which the radial-orthoradial frame $F=u_{1} F_{r}+u_{2} F_{\text {or }}+u_{3} F_{c}$, where $F_{r}=\frac{q}{r} \frac{\partial}{\partial \dot{q}}, F_{c}=\frac{c}{c} \frac{\partial}{\partial \dot{q}}$, and $F_{\text {or }}=F_{c} \times F_{r}$. The minimization of the transfer time is a relevant issue since the power of the engine is limited by the constraint on the control $|F|=\left(u_{1}^{2}+u_{2}^{2}+u_{3}^{2}\right)^{1 / 2} \leq F_{\max }$. Instead of position-speed cartesian coordinates, we use in practice coordinates that give an account of the geometry of the trajectory, namely the orbit elements: we set $x=(P, e, h, l)$ where $P$ is the semi-latus rectum of the osculating ellipse, $e=\left(e_{x}, e_{y}\right)$ the eccentricity vector, $h=\left(h_{x}, h_{y}\right)$ the inclination vector, and $l$ the cumulated longitude. The associated equations of motion are the Gauss equations [29] and, in these coordinates, $\dot{x}=F_{0}+\left(u_{1} F_{r}+u_{2} F_{\text {or }}+u_{3} F_{c}\right) / m$, with

$$
\begin{aligned}
F_{0} & =\sqrt{\frac{\mu}{P}} \frac{W^{2}}{P} \frac{\partial}{\partial l}, \quad F_{r}=\frac{1}{W} \sqrt{\frac{P}{\mu}}\left(-W \sin l \frac{\partial}{\partial e_{x}}+W \cos l \frac{\partial}{\partial e_{y}}\right), \\
F_{\text {or }} & =\frac{1}{W} \sqrt{\frac{P}{\mu}}\left(2 P \frac{\partial}{\partial P}+\left(W \cos l+\eta_{x}\right) \frac{\partial}{\partial e_{x}}+\left(W \sin l+\eta_{y}\right) \frac{\partial}{\partial e_{y}}\right), \\
F_{c} & =\frac{1}{W} \sqrt{\frac{P}{\mu}}\left(-Z e_{y} \frac{\partial}{\partial e_{x}}+Z e_{x} \frac{\partial}{\partial e_{y}}+\frac{C \cos l}{2} \frac{\partial}{\partial h_{x}}+\frac{C \sin l}{2} \frac{\partial}{\partial h_{y}}+Z \frac{\partial}{\partial l}\right) .
\end{aligned}
$$

Hereabove, $W=1+e_{x} \cos l+e_{y} \sin l, Z=h_{x} \sin l-h_{y} \cos l, \eta_{x}=e_{x}+\cos l, \eta_{y}=e_{y}+\sin l$, and $C=1+h^{2}$.

\section{Regular extremals}

The extremals of the problem are parametrized by the maximum principle. Since the final mass is free, the maximization and transversality conditions imply that $|F|=F_{\max }$ almost everywhere [14], so the mass equation can be eliminated: $m(t)=m_{0}-\beta F_{\max } t$, and the system becomes a non-autonomous sub-Riemannian system with drift [9]. We consider the normal case and normalize as before the cost multiplier by $p^{0}=-1$. The resulting Hamiltonian is $H=-1+H_{0}+\left(u_{1} H_{1}+u_{2} H_{2}+u_{3} H_{3}\right) / m(t)$, where $H_{1}, H_{2}$ and $H_{3}$ are the Hamiltonian lifts of the corresponding vector fields $\left(H_{1}=\left\langle p, F_{r}\right\rangle\right.$, etc). The switching surface is $\Sigma=\left\{H_{1}=\right.$ $\left.H_{2}=H_{3}=0\right\}$ and the extremals are classified by the order of their contact with $\Sigma$ [10]: as in (12), the order zero extremals are then defined as the integral curves of the reduced Hamiltonian (smooth on $T^{*} M \backslash \Sigma$ ), given by $H_{r}=H_{0}+\frac{F_{\max }}{m(t)}\left(H_{1}^{2}+H_{2}^{2}+H_{3}^{2}\right)^{1 / 2}$, and contained in the level set $\left\{H_{r}=0\right\}$. In order to see that these extremals are regular in the sense of the previous section, one may reparametrize the control $u_{r}=$ $F_{\max }\left(H_{1}, H_{2}, H_{3}\right) / \sqrt{H_{1}^{2}+H_{2}^{2}+H_{3}^{2}}$ by $u_{r}=F_{\max }(\sin \varphi \cos \theta, \sin \varphi \sin \theta, \cos \varphi),(\theta, \varphi)$ in $\mathbb{R}^{2}$. Actually, one has the following result (see [12]). 
Proposition 4.1. The extremals of order zero are smooth responses to smooth controls on the boundary of $|u| \leq F_{\max }$. They are singularities of the endpoint mapping $E_{x_{0}, t_{f}}: u \mapsto x\left(t_{f}, x_{0}, u\right)$ for the $L^{\infty}$-topology when $u$ is restricted to the sphere $F_{\max } \mathbb{S}^{2}$.

Since our aim is to check the optimality status of smooth extremals, we restrict ourselves to the order zero case, not taking into account possibly broken extremals arising as concatenation of order zero arcs. Indeed, the Lie structure of the vector fields $F_{0}, \ldots, F_{c}$ imply that the only possible contacts with $\Sigma$ are of order one: at such a point, the control rotates instantaneously of an angle $\pi$ and the singularity is called a П-singularity [12]. These extremals cross $\Sigma$ with a given slope and are not difficult to deal with numerically. Moreover, having only order one contacts also means that there are no singular trajectories on the sphere: the order zero extremals are the relevant smooth objects to analyze here (see [12] for the analysis of an exceptional singular in the case of a single-input $2 \mathrm{D}$ orbit transfer).

\section{Conjugate points}

In accordance with Section 2.3, the exponential mapping is defined on the codimension one (if nonempty) subset $X=\left\{p_{0} \in T_{x_{0}}^{*} M \mid H_{r}\left(0, x_{0}, p_{0}\right)=0\right\}$ by $\exp _{x_{0}, t}\left(p_{0}\right)=x\left(t, x_{0}, p_{0}\right)$, and the shooting function is $S\left(t_{f}, p_{0}\right)=$ $\exp _{x_{0}, t_{f}}\left(p_{0}\right)-x_{f}$, where $x_{f}$ is the prescribed terminal point. The extremals we are intersted in are defined by the zeros of this function. The mapping $S$ is smooth outside the set $\Pi$ of points $p_{0}$ generating $\Pi$-singularities. The behavior of $S$ at such points can be made precise using a nilpotent model of the dynamics (see [9]): in this model, the set $\Pi$ can be stratified into subsets of codimension greater than one in the domain $X$ of $\exp _{x_{0}, t}$. Thus, $\Pi$-singularities are not a crucial issue when solving for $S\left(t_{f}, p_{0}\right)=0$ numerically by means of Newton-like methods. As explained in the normal case with free final time, the time $t_{c}$ is conjugate if and only if

$$
\operatorname{rank}\left\{\delta x_{1}\left(t_{c}\right), \ldots, \delta x_{n-1}\left(t_{c}\right)\right\}<n-1,
$$

where $\delta z_{i}=\left(\delta x_{i}, \delta p_{i}\right)$ are the Jacobi fields solution of the linearized system $\delta \dot{z}=\mathrm{d} \vec{H}_{r}(t, z(t)) \cdot \delta z$ along the extremal $z=(x, p)$, with initial conditions $\delta z_{i}(0)$ such that $\delta p_{1}(0), \ldots, \delta p_{n-1}(0) \operatorname{span} T_{p(0)} X$,

$$
\frac{\partial H_{r}}{\partial p}\left(0, x_{0}, p(0)\right) \cdot \delta p_{i}(0)=0, i=1, \ldots, n-1 .
$$

Remark 4.2. In the minimal time problem, normal case, as an alternative to $p^{0}=-1$ one can normalize the adjoint covector by letting $p(0)$ belong to the projective space $\mathbb{P}^{n-1}(\mathbb{R})$, or to $\mathbb{S}^{n-1}$. In both cases, $p(0)$ is oriented by the condition $p^{0}<0$. Hence, instead of (28), one can also let the initial Jacobi fields be defined by $\delta p_{i}(0) \perp p(0), i=1, \ldots, n-1$.

The rank condition (27) is checked by performing a singular value decomposition of $\delta x_{1}\left(t_{c}\right), \ldots, \delta x_{n-1}(t)$. An equivalent and complementary test is Test 3 of Section 2.3, namely, $\delta x_{1}\left(t_{c}\right) \wedge \cdots \wedge \delta x_{n-1}\left(t_{c}\right) \wedge \dot{x}\left(t_{c}\right)=0$. Indeed, this second test allows us to look for a sign change in the determinant. In practice, a coarse dichotomy procedure is used so as to locate the conjugate times and then initialize a finer Newton method. Since the determinant has roughly the same magnitude as the product of the singular values associated to the rank (27), it is relevant numerically to extract only the smallest singular values which already contain the complete rank information. A simulation is presented for $F_{\max }=6$ Newton. The boundary conditions defining the initial and terminal orbits are provided in Table 2 (observe that the cumulated longitude is fixed: the problem is to minimize the time for a prescribed number of revolutions around the Earth). A time minimizing extremal is analyzed at Figures 5 and 6.

We conclude this section by a preliminary computation of a subset of the conjugate locus $C\left(x_{0}\right)$ of the initial orbit. By definition, $C\left(x_{0}\right)$ is the set of first conjugate points on extremals starting from $x_{0}$. The first simulation in Figure 7 represents the conjugate points along extremals generated by making variations of the final cumulated longitude of the target: $l$ in $[51,51+2 \pi]$. This simulation is then completed in Figure 8 by making variations on the eccentricity of the same geostationnary target: $l$ is now prescribed to 51 while $e_{x}$ 

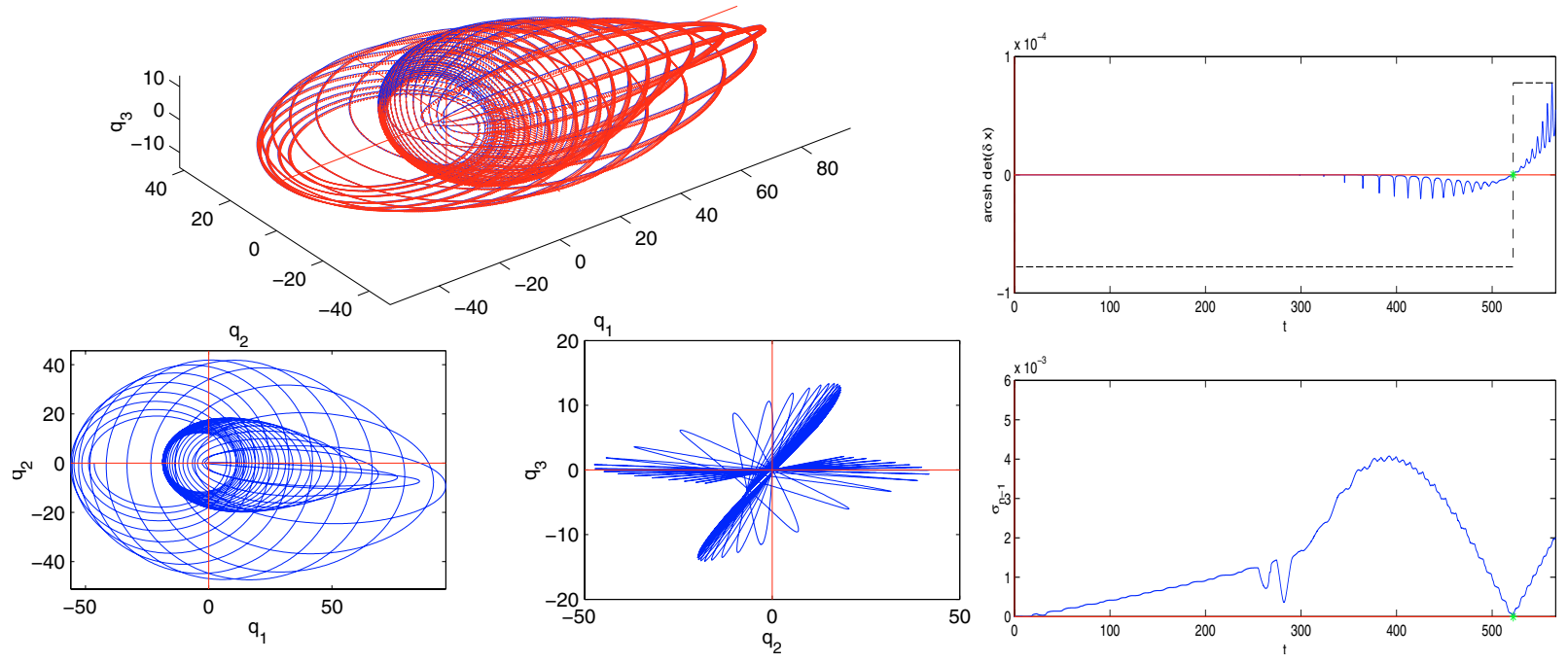

Figure 5. A 6 Newton transfer. The minimum time is $t_{f} \simeq 141.60$ hours (about 6 days) for 7 revolutions around the Earth, approximatively. On the left, the optimal trajectory (with projections in the equatorial plane and a perpendicular plane to illustrate how the inclination is corrected) is extended until 4 times the minimum time. Up right, the determinant, bottom right, the smallest singular value of the Jacobi fields associated to the extremal. The first conjugate time $t_{c}$ is detected about 3.7 times the minimum time: the (local) time optimality of the trajectory is lost after $t_{c} \simeq 522.07$ hours.

TABLE 2. Boundary conditions.

\begin{tabular}{lll}
\hline Variable & Initial cond. & Final cond. \\
\hline$P$ & $11.625 \mathrm{Mm}$ & $42.165 \mathrm{Mm}$ \\
$e_{x}$ & 0.75 & 0 \\
$e_{y}$ & 0 & 0 \\
$h_{x}$ & 0.0612 & 0 \\
$h_{y}$ & 0 & 0 \\
$l$ & $\pi \mathrm{rad}$ & $51 \mathrm{rad}$ \\
\hline
\end{tabular}

and $e_{y}$ vary in $[-3 e-2,3 e-2]$. This computation is a first step towards obtaining the cut locus, namely the set of points where the global optimality is lost ${ }^{4}$. The ultimate goal is the possibility to determine the optimal strategy from the knowledge of the cut locus.

All the simulations presented here are based on the COTCOT code, and reproducible using sources available at www.n7.fr/apo/cotcot.

\subsection{Minimal time attitude control}

\section{Euler equations}

The equations of the problem are those of a rigid body rotating around its center of gravity under the action of central torques. Let $O$ be the center of gravity of the spacecraft. Consider the two sets of orthonormal frames

\footnotetext{
${ }^{4}$ In some cases, e.g., the Riemannian case, the loss of global optimality occurs either at a conjugate point or at a point where two minimizing geodesics having the same length intersect themselves. Morever, on the sphere of dimension two, in the real analytic case, the cut is a tree whose extremities are conjugate points, conjugate-cut points being cusps of the conjugate locus (see [17]).
} 

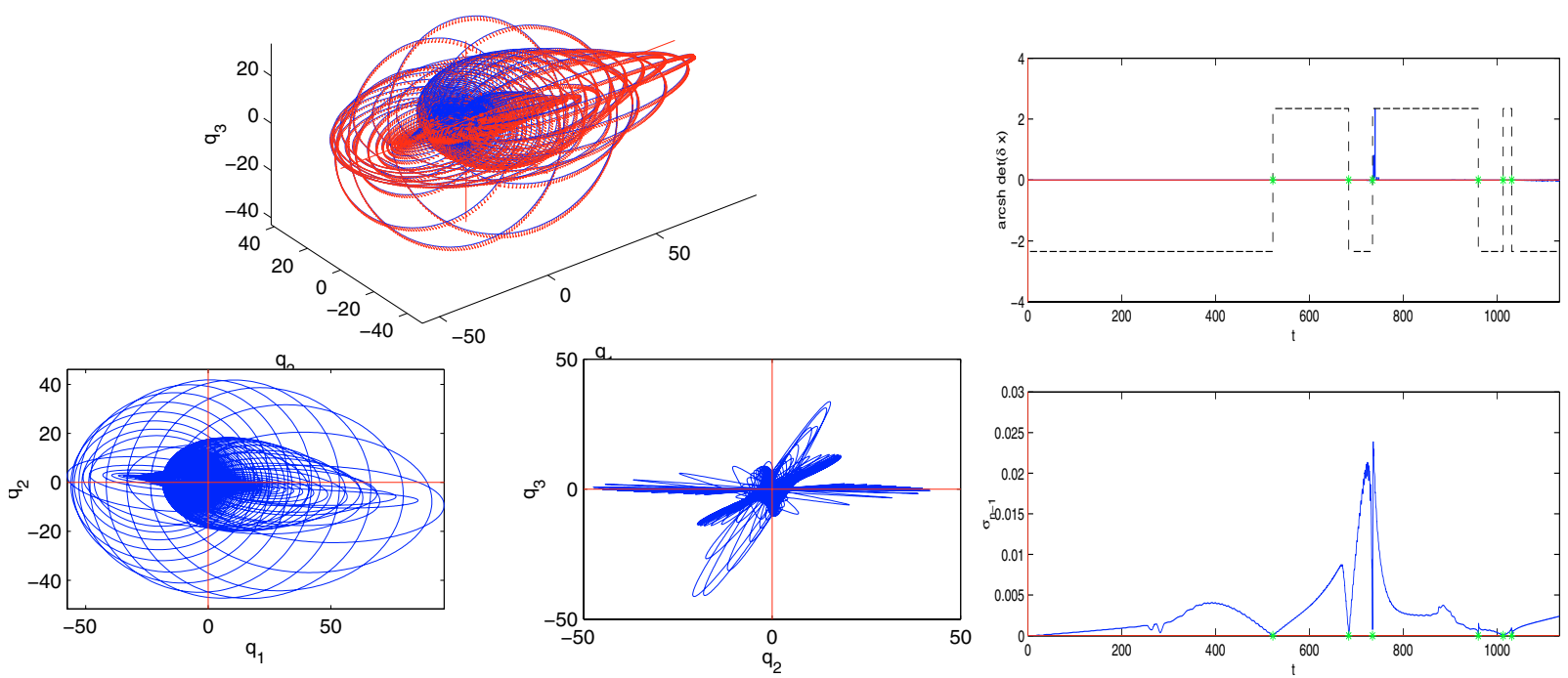

FiguRE 6. The same transfer extended 8 times: six conjugate points are detected.
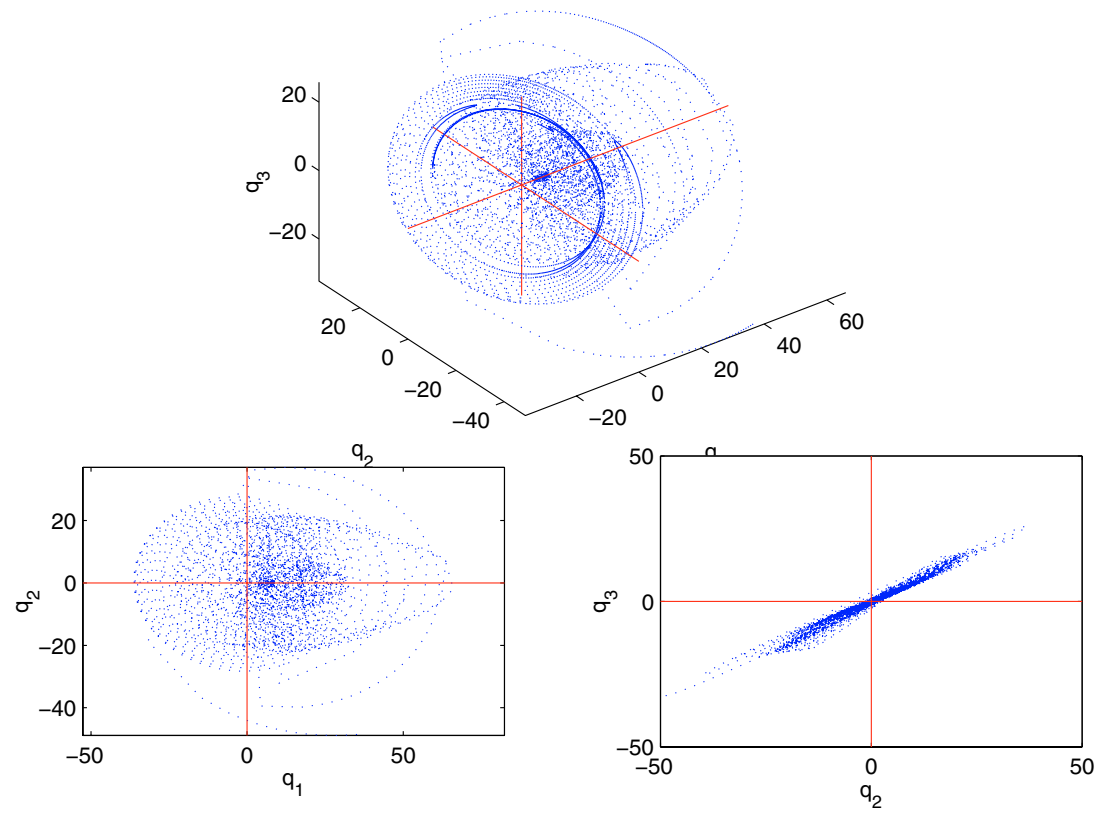

Figure 7. Conjugate points, $F_{\max }=6$ Newton. The initial orbit and longitude are as defined in Table 2. The cumulated longitude on the final geostationnary orbit is taken in $[51,51+2 \pi]$. A uniform discretization of $1 e 3$ points is considered.

with common origin $O: s=\left(e_{1}, e_{2}, e_{3}\right)$, and $a=\left(E_{1}, E_{2}, E_{3}\right)$. Let $s$ be fixed inertial space, and $a$ be fixed in the spacecraft, $E_{i}$ being the principal axes of the rigid body. The attitude of the spacecraft is given by the matrix of direction cosines $R(t)=\left(r_{i j}(t)\right) \in S O(3)$, where $r_{i j}(t)=\left\langle E_{i}(t), e_{j}\right\rangle$. The evolution of $R(t)$ is described by the matrix equations

$$
\dot{R}(t)=S(\Omega(t)) R(t)
$$



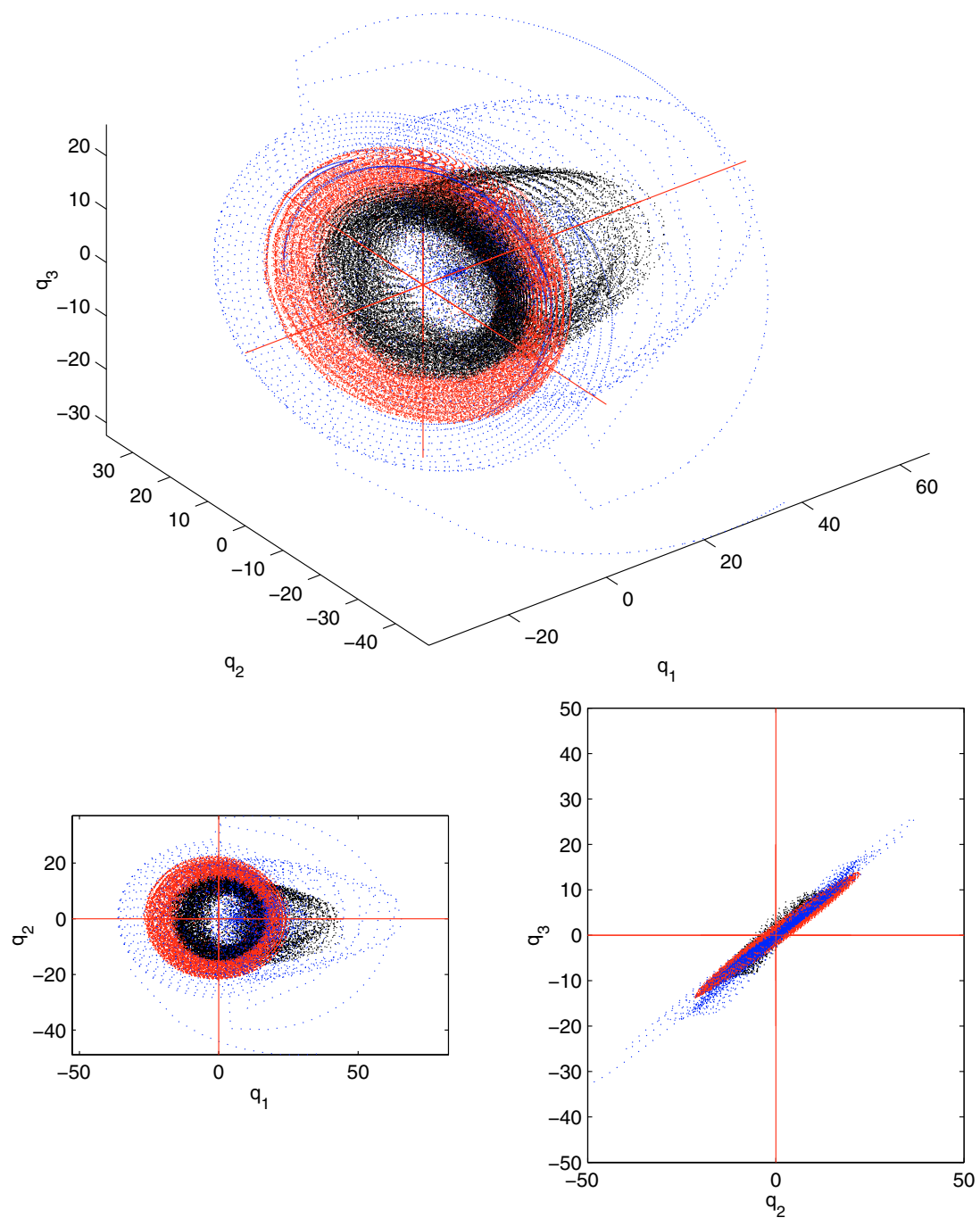

Figure 8. Conjugate points. Those coming from the variations of the longitude are in blue (see Fig. 7). Those due to variations on the eccentricity are in black (negative $e_{x}$ ) and red (positive $e_{x}$ ). A uniform discretization of $1 e 4$ points is considered.

where

$$
S(\Omega)=\left(\begin{array}{ccc}
0 & \Omega_{3} & -\Omega_{2} \\
-\Omega_{3} & 0 & \Omega_{1} \\
\Omega_{2} & -\Omega_{1} & 0
\end{array}\right)
$$

and $\Omega(t)=\left(\Omega_{1}, \Omega_{2}, \Omega_{3}\right)^{T}$ is the angular velocity with respect to the moving frame $a$.

The equations describing the motion of $\Omega(t)$ are the Euler equations

$$
\dot{\Omega}_{1}=a_{1} \Omega_{2} \Omega_{3}+b_{1} u, \quad \dot{\Omega}_{2}=a_{2} \Omega_{1} \Omega_{3}+b_{2} u, \quad \dot{\Omega}_{3}=a_{3} \Omega_{1} \Omega_{2}+b_{3} u,
$$

where $a_{1}=\frac{I_{2}-I_{3}}{I_{1}}, a_{2}=\frac{I_{3}-I_{1}}{I_{2}}, a_{3}=\frac{I_{1}-I_{2}}{I_{3}}$, and where $I_{1}, I_{2}$, and $I_{3}$ are the principal moments of inertia of the body, assumed to be such that $I_{1}>I_{2}>I_{3}>0$, and $b=\left(b_{1}, b_{2}, b_{3}\right)^{T}$ is a fixed vector of $\mathbb{R}^{3}$. 

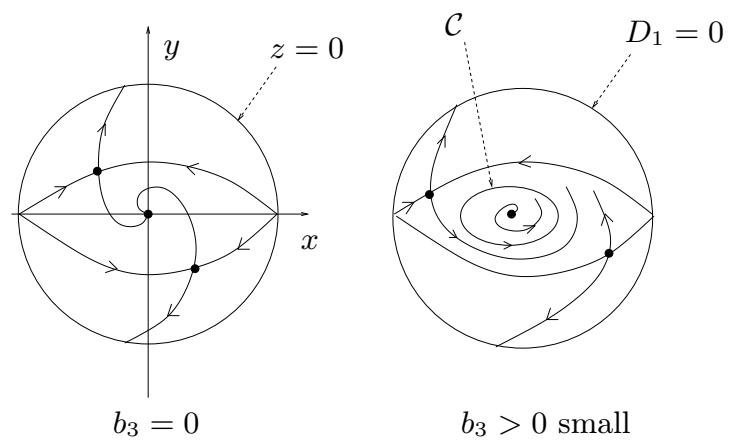

Figure 9. Topological behavior in function of $b_{3}$.

The control torque is applied by a pair of opposite gas jets. The vector $\left(I_{1} b_{1}, I_{2} b_{2}, I_{3} b_{3}\right)$ represents the direction along which the control torque is applied with respect to the body axes $a$.

Up to changing coordinates, we assume that $a_{1}=a_{3}=1, a_{2}=-1$.

\section{Singular trajectories (subsystem)}

We restrict ourselves to the system of Euler equations (30). The system has the form $\dot{q}(t)=f_{0}(q(t))+$ $u(t) f_{1}(q(t))$, where $q(t)=(x(t), y(t), z(t))^{T} \in \mathbb{R}^{3}$. Let $q(\cdot)$ be a singular trajectory of that system, associated to a control $u_{s}$. We assume that $f_{1}$ and $\left[f_{1}, f_{0}\right]$ to be linearly independent. The first order Pontryagin cone is $K=\operatorname{Span}\left\{f_{1},\left[f_{1}, f_{0}\right]\right\}$. The adjoint vector satisfies $\left\langle p, f_{1}\right\rangle=\left\langle p,\left[f_{0}, f_{1}\right]\right\rangle=0$ along $q(\cdot)$, and thus, if the vector fields $f_{0},\left[f_{1}, f_{0}\right]$, and $\left[\left[f_{1}, f_{0}\right], f_{1}\right]$, are linearly independent, then the singular control $u_{s}$ is given in the feedback form by $u_{s}(q)=-\frac{D_{2}(q)}{D_{1}(q)}$, where $D_{1}(q)=\operatorname{det}\left(f_{1},\left[f_{1}, f_{0}\right],\left[\left[f_{1}, f_{0}\right], f_{1}\right]\right)$, and $D_{2}(q)=\operatorname{det}\left(f_{1},\left[f_{1}, f_{0}\right],\left[\left[f_{1}, f_{0}\right], f_{0}\right]\right)$. Hence, singular trajectories are solutions of the differential equation $\dot{q}(t)=X_{s}(q(t))$ in $\mathbb{R}^{3}$, where $X_{s}(q)=$ $f_{0}(q)-\frac{D_{2}(q)}{D_{1}(q)} f_{1}(q)$. The exceptional singular trajectories are contained in the invariant set $D_{3}=0$, where $D_{3}(q)=\operatorname{det}\left(f_{1},\left[f_{1}, f_{0}\right], f_{0}\right)$, and from Remark 2.28 , there exists no conjugate time along such trajectories. The hyperbolic (resp., elliptic) singular trajectories are contained in $D_{1} D_{3}>0$ (resp., $\left.D_{1} D_{3}<0\right)$.

In order to compute numerically conjugate times along hyperbolic or elliptic singular trajectories, we apply the algorithm described previously by Test 2 (see Eqs. (23)). This amounts to computing a unique Jacobi field, and testing collinearity with the vector field $f_{1}$, at initial and final times.

In the case of Euler equations, there holds

$$
\begin{aligned}
D_{1}= & 2 a_{1} b_{2} b_{3}\left(a_{3} b_{2}^{2}-a_{2} b_{3}^{2}\right) \Omega_{1}+2 a_{2} b_{1} b_{3}\left(a_{1} b_{3}^{2}-a_{3} b_{1}^{2}\right) \Omega_{2}+2 a_{3} b_{1} b_{2}\left(a_{2} b_{1}^{2}-a_{1} b_{2}^{2}\right) \Omega_{3}, \\
D_{2}= & a_{3}^{2} b_{1} b_{2}\left(b_{1} \Omega_{2}+b_{2} \Omega_{1}\right)\left(a_{2} \Omega_{1}^{2}-a_{1} \Omega_{2}^{2}\right)+a_{1}^{2} b_{2} b_{3}\left(b_{2} \Omega_{3}+b_{3} \Omega_{2}\right)\left(a_{3} \Omega_{2}^{2}-a_{2} \Omega_{3}^{2}\right) \\
& +a_{2}^{2} b_{1} b_{3}\left(b_{1} \Omega_{3}+b_{3} \Omega_{1}\right)\left(a_{1} \Omega_{3}^{2}-a_{3} \Omega_{1}^{2}\right) \\
D_{3}= & a_{2} a_{3} b_{1} \Omega_{1}^{2}\left(b_{2} \Omega_{3}-b_{3} \Omega_{2}\right)+a_{1} a_{3} b_{2} \Omega_{2}^{2}\left(b_{3} \Omega_{1}-b_{1} \Omega_{3}\right)+a_{1} a_{2} b_{3} \Omega_{3}^{2}\left(b_{1} \Omega_{2}-b_{2} \Omega_{1}\right) .
\end{aligned}
$$

The topological behavior of singular trajectories depends on the choice of $b$ (see [8]).

Numerical simulations. For the numerical simulations, we choose $a_{1}=1, a_{2}=-1, a_{3}=1, b_{1}=2, b_{2}=1$, and $b_{3}$ is a small parameter acting on the topological behavior. If $b_{3}=0$, then the set $D_{3}=0$ is the union of the plane $\Omega_{3}=0$ and the line $\Omega_{1}=\Omega_{2}=0$.

In Figure 9, we represent the projection of singular trajectories on the unit sphere $S^{2}$ of $\mathbb{R}^{3}$. The equator corresponds to the plane $D_{1}=0$. If $b_{3}=0$, all singular trajectories are hyperbolic, except one singular trajectory that projects onto the point defined by the intersection of the line $x=y=0$ with the sphere $S^{2}$. If $b_{3}>0$ is small, then there appears a limit cycle $\mathcal{C}$, corresponding to one branch of the algebraic set $D_{3}=0$. Inside $\mathcal{C}$, we 

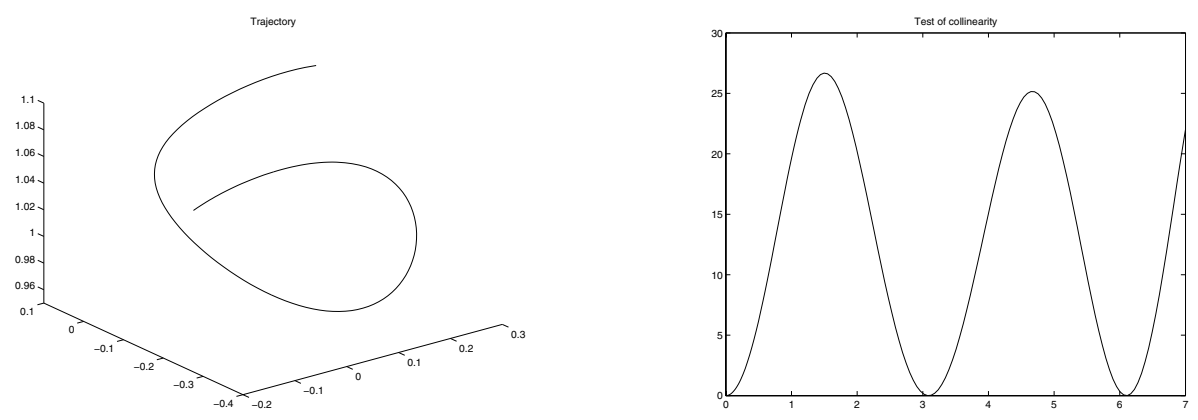

Figure 10. Numerical results for Euler equations, hyperbolic case.
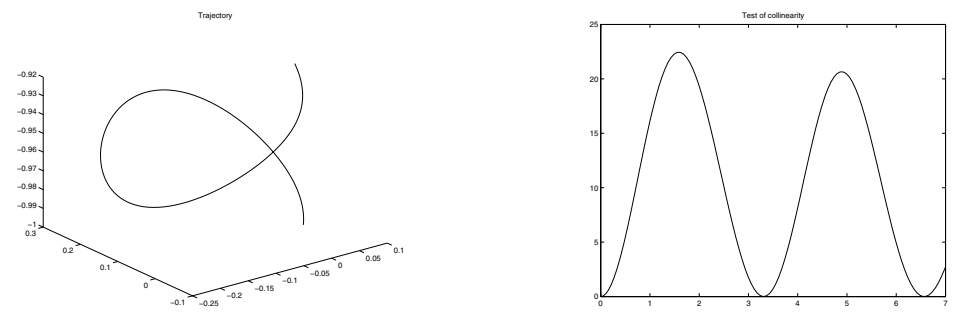

Figure 11. Numerical results for Euler equations, elliptic case.

have projections of elliptic singular trajectories, and outside, and close to $\mathcal{C}$, we have projections of hyperbolic singular trajectories.

The numerical test is achieved with the initial data $\Omega_{1}(0)=0.05, \Omega_{2}(0)=0.05$, and $b_{3}=0.3$. Solving $D_{3}=0$ with respect to $\Omega_{3}$, in the neighborhood of 0 , one gets $\Omega_{3}=0.0112$ around. The trajectory corresponding to $\Omega_{3}(0)=0.0112$ is exceptional, and has no conjugate time. We observe numerically that trajectories having an initial condition $\Omega_{3}(0)$ close to 0.0112 have no conjugate time. This is in accordance with our previous results.

In contrast, in Figure 10 (resp., in Fig. 11), are represented an hyperbolic trajectory, with $\Omega_{3}(0)=1$ (resp., an elliptic trajectory, with $\Omega_{3}(0)=-1$ ), whose projection converges to the limit cycle $\mathcal{C}$, and having conjugate times. The zeros of the curve on the right-hand side of the figure represent the conjugate times (test of collinearity).

\section{Singular trajectories (complete system)}

The complete system of attitude control is given by equations (29) and (30). To compute conjugate times, in order to illustrate both algorithms described previously, we choose here the method of the integral transformation (Test 1 ). The vector field $f_{1}$ being constant, one just has to achieve a linear change of coordinates. More precisely, assuming $b_{3} \neq 0$, we achieve the integral transformation by considering as a new control the control $v=x_{3}$, and we define the new coordinates $x=\Omega_{1}-\frac{b_{1}}{b_{3}} \Omega_{3}, y=\Omega_{2}-\frac{b_{2}}{b_{3}} \Omega_{3}$. The reduced system writes

$$
\dot{R}(t)=S(x(t), y(t), v(t)) R(t), \quad \dot{x}(t)=f_{1}(x(t), y(t), v(t)), \quad \dot{y}(t)=f_{2}(x(t), y(t), v(t)),
$$

where $f_{1}$ and $f_{2}$ are quadratic.

For the numerical simulations, the initial data on the state (that is an element of $\mathbb{R}^{11}$ ) are $R(0)=I d, x(0)=$ $0.05, y(0)=0.05$.

Hyperbolic case. If we choose the initial adjoint vector $p(0)=\left(\begin{array}{llllllllllll}1 & 1 & 1 & 1 & 1 & 1 & 1 & 1 & 1 & 1 & 1\end{array}\right)$, then we are in the hyperbolic case. In order to compute the rank of the matrix, we use a singular value decomposition (SVD). We observe that, except at a conjugate time, the rank of this matrix is equal to 4 . Figure 12 represents the singular 

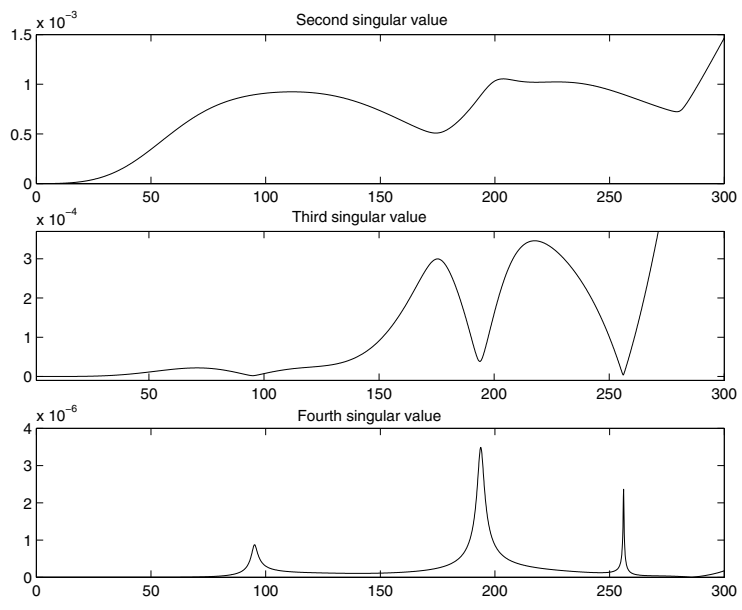

FIGURE 12. Numerical results on attitude control, hyperbolic case.
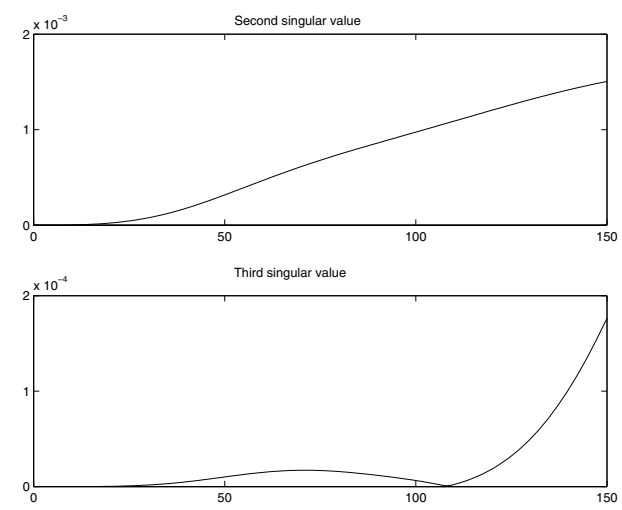

FIGURE 13. Numerical results on attitude control, exceptional case.

values 2,3 and 4, and the first conjugate time corresponds to the vanishing of the fourth singular value. We get $t_{c} \simeq 285.729$.

Exceptional case. If we choose the initial adjoint vector $p(0)=\left(\begin{array}{llllll}1 & 0.9935541287639311111 & 1 & 1 & 1\end{array}\right)$, then we are in the exceptional case. To compute the rank of the matrix, we also use a singular value decomposition (SVD). We observe that, except at a conjugate time, the rank of this matrix is equal to 3. Figure 13 represents the singular values 2 and 3, and the first conjugate time corresponds to the vanishing of the third singular value. We get $t_{c c} \simeq 108.1318$.

Remark 4.3. We can observe in Figure 12 that the third singular value almost vanishes for $t \simeq 100$. This is due to the fact that the hyperbolic extremal we chose is not so far from the exceptional one, simulated in Figure 13; since the singular values depend continuously on $p(0)$, when $p(0)$ passes from an hyperbolic to the exceptional case then the third singular value vanishes.

Remark 4.4. In the exceptional case, it is also interesting to compute the time $t_{c}$. Numerically, it corresponds to the second time at which the third singular value vanishes, and we obtain $t_{c} \simeq 327.5$. 


\section{REFERENCES}

[1] A.A. Agrachev and R.V. Gamkrelidze, Second order optimality condition for the time optimal problem. Matem. Sbornik 100 (1976) 610-643. English transl. in: Math. USSR Sbornik 29 (1976) 547-576.

[2] A.A. Agrachev and R.V. Gamkrelidze, Symplectic geometry for optimal control, Nonlinear controllability and optimal control. Dekker, New York, Monogr. Textbooks Pure Appl. Math. 133 (1990) 263-277.

[3] A.A. Agrachev and Yu.L. Sachkov, Control theory from the geometric viewpoint, Encyclopedia of Mathematical Sciences, 87. Control Theory and Optimization, II. Springer-Verlag, Berlin (2004) 412 pp.

[4] A.A. Agrachev and A.V. Sarychev, Abnormal sub-Riemannian geodesics: Morse index and rigidity. Ann. Inst. Henri Poincaré 13 (1996) 635-690.

[5] A.A. Agrachev and A.V. Sarychev, On abnormal extremals for Lagrange variational problems. J. Math. Syst. Estim. Cont. 8 (1998) 87-118.

[6] C. Bischof, A. Carle, P. Kladem and A. Mauer, Adifor 2.0: Automatic Differentiation of Fortran 77 Programs. IEEE Comput. Sci. Engrg. 3 (1996) 18-32.

[7] O. Bolza, Calculus of variations. Chelsea Publishing Co., New York (1973).

[8] B. Bonnard, Feedback equivalence for nonlinear systems and the time optimal control problem. SIAM J. Control Optim. 29 (1991) 1300-1321.

[9] B. Bonnard and J.-B. Caillau, Introduction to nonlinear optimal control, in Advances Topics in Control Systems Theory, Lecture Notes from FAP 2004, F. Lamnabhi-Lagarrigue, A. Loria, E. Panteley Eds., Springer, Berlin (2005).

[10] B. Bonnard and M. Chyba, The role of singular trajectories in control theory. Springer Verlag, New York (2003).

[11] B. Bonnard and I. Kupka, Théorie des singularités de l'application entrée/sortie et optimalité des trajectoires singulières dans le problème du temps minimal. Forum Math. 5 (1993) 111-159.

[12] B. Bonnard, J.-B. Caillau and E. Trélat, Geometric optimal control of elliptic Keplerian orbits. Discrete Contin. Dyn. Syst. 5 (2005) 929-956.

[13] B. Bonnard, J.-B. Caillau and E. Trélat, Cotcot: short reference manual, ENSEEIHT-IRIT Technical Report RT/APO/05/1 (2005) www.n7.fr/apo/cotcot.

[14] J.B. Caillau, J. Noailles and J. Gergaud, 3D Geosynchronous Transfer of a Satellite: Continuation on the Thrust. J. Opt. Theory Appl. 118 (2003) 541-565.

[15] Y. Chitour, F. Jean and E. Trélat, Genericity results for singular trajectories. J. Diff. Geom. 73 (2006) 45-73.

[16] J. de Morant, Contrôle en temps minimal des réacteurs chimiques discontinus. Ph.D. Thesis, Univ. Rouen (1992).

[17] S. Galot, D. Hulin and J. Lafontaine, Riemannian geometry. Springer-Verlag, Berlin (1987).

[18] B.S. Goh, Necessary conditions for singular extremals involving multiple control variables. SIAM J. Cont. 4 (1966) $716-731$.

[19] M.R. Hestenes, Application of the theory of quadratic forms in Hilbert spaces to the calculus of variations. Pac. J. Math. 1 (1951) 525-582.

[20] M.R. Hestenes, Optimization theory - the finite dimensional case. Wiley (1975).

[21] A.D. Ioffe and V.M. Tikhomirov, Theory of extremal problems. North-Holland Publishing Co., Amsterdam (1979).

[22] H.J. Kelley, R. Kopp and H.G. Moyer, Singular extremals, in Topics in optimization, G. Leitman Ed., Academic Press, New York (1967) 63-101.

[23] A.J. Krener, The high-order maximum principle and its applications to singular extremals. SIAM J. Cont. Opt. 15 (1977) 256-293.

[24] L. Pontryagin, V. Boltyanskii, R. Gamkrelidze and E. Mischenko, The mathematical theory of optimal processes. Wiley Interscience (1962).

[25] A.V. Sarychev, The index of second variation of a control system. Matem. Sbornik 113 (1980) 464-486. English transl. in: Math. USSR Sbornik 41 (1982) 383-401.

[26] L.F. Shampine, H.A. Watts and S. Davenport, Solving non-stiff ordinary differential equations - the state of the art. Technical Report sand75-0182, Sandia Laboratories, Albuquerque, New Mexico (1975).

[27] E. Trélat, Asymptotics of accessibility sets along an abnormal trajectory. ESAIM: COCV 6 (2001) 387-414.

[28] L.C. Young, Lectures on the calculus of variations and optimal control theory. Chelsea, New York (1980).

[29] O. Zarrouati, Trajectoires spatiales. CNES-Cepadues, Toulouse (1987). 